\title{
Gradual SEP Events
}

\begin{abstract}
Gradual solar energetic-particle (SEP) events are "big proton events" and are usually much more "gradual" in their decay than in their onset. As their intensities increase, particles streaming away from the shock amplify Alfvén waves that scatter subsequent particles, increasing their acceleration, eventually limiting ion flow at the "streaming limit." Waves generated by higher-speed protons running ahead can also throttle the flow of lower-energy ions, flattening spectra and altering abundances in the biggest SEP events. Thus, we find that the $A / Q$ dependence of scattering causes element-abundance patterns varying in space and time, which define source-plasma temperatures $T$, since the pattern of $Q$ values of the ions depends upon temperature. Differences in $T$ explain much of the variation of element abundances in gradual SEP events. In nearly $70 \%$ of gradual events, SEPs are shock-accelerated from ambient coronal plasma of $\sim 0.8-1.6 \mathrm{MK}$, while $24 \%$ of the events involve material with $T \approx 2-4 \mathrm{MK}$ re-accelerated from residual impulsive-suprathermal ions with pre-enhanced abundances. This source-plasma temperature can occasionally vary with solar longitude across the face of a shock. Non-thermal variations in ion abundances in gradual SEP events reaccelerated from the 2-4 MK impulsive source plasma are reduced, relative to those in the original impulsive SEPs, probably because the accelerating shock waves sample a pool of ions from multiple jet sources. Late in gradual events, SEPs become magnetically trapped in a reservoir behind the CME where spectra are uniform in space and decrease adiabatically in time as the magnetic bottle containing them slowly expands. Finally, we find variations of the $\mathrm{He} / \mathrm{O}$ abundance ratio in the source plasma of different events.
\end{abstract}

We begin by showing proton intensities in the classic large gradual SEP event of 4 November 2001 in Fig. 5.1. This event, from a source longitude of W17 on the Sun, has the typical time profile of a centrally located event (see Sect. 2.3.3). The 


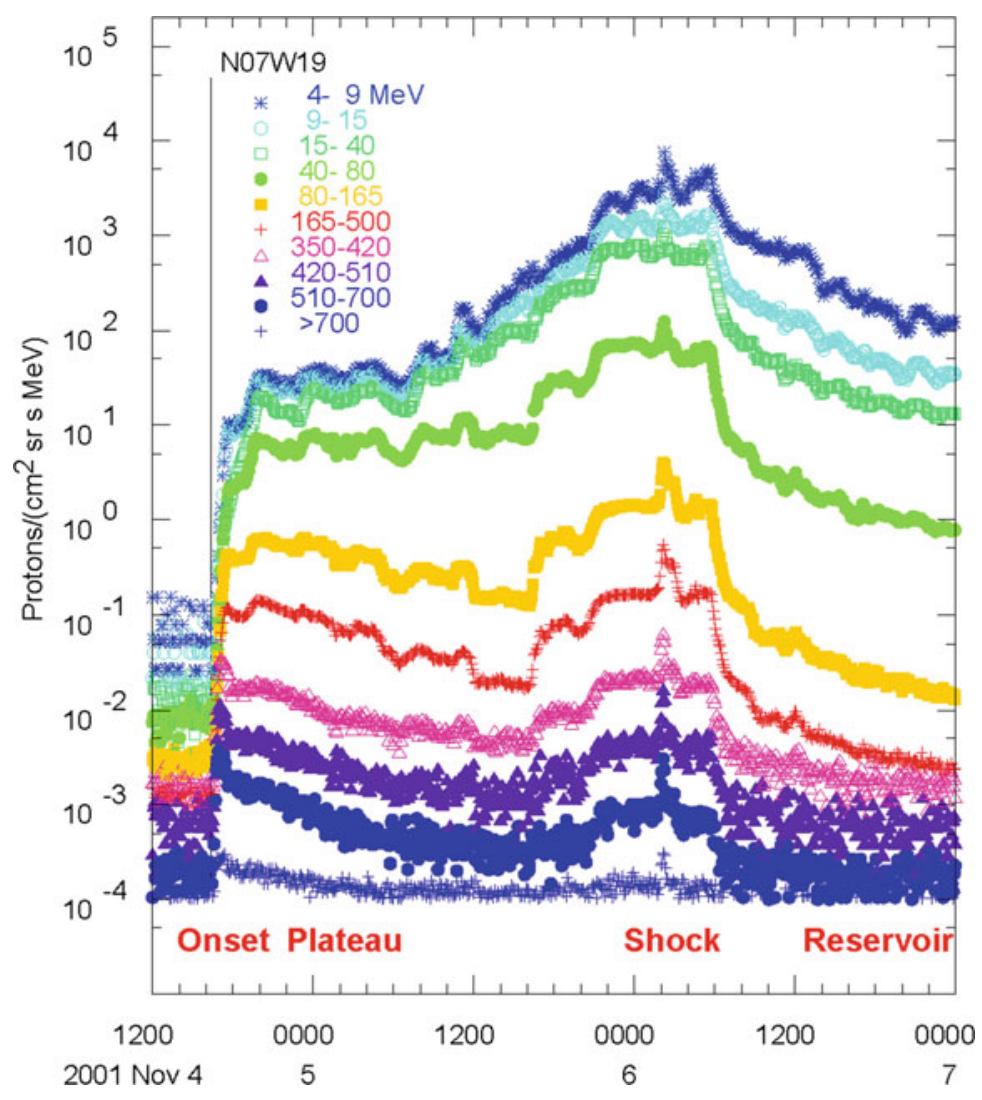

Fig. 5.1 Proton intensities vs. time from the NOAA/GOES satellite are shown for the large gradual SEP event of 4 November 2001 at solar longitude W19 (compare Fig. 2.2). Distinctive event phases are listed along the abscissa (Reames 2013 (C) Springer)

figure lists phases of the event along the abscissa, which we will study in approximate time order, although onsets were discussed previously in Sect. 3.1.

In impulsive SEP events, most particles traveled to us scatter free so we had little need to discuss transport. With increased intensities, protons from gradual SEP events generate or amplify their own spectrum of resonant Alfvén waves for pitchangle scattering, which complicates their transport more and more as intensities increase. In fact, it is the resonant waves, generated by the out-flowing particles, which scatter subsequent particles back and forth across the shock, incrementally increasing ion velocity, driving particles to higher and higher energy.

For recent reviews of gradual SEP events see Desai and Giacalone (2016), and Lee et al. (2012). For theoretical background see Parker (1963) and Jones and Ellison (1991). 


\subsection{Parallel Transport}

\subsubsection{Diffusive Transport}

The diffusion of particles of type $\mathrm{X}$ and velocity $v$ by pitch-angle scattering with scattering mean free path $\lambda_{X}$ with a power-law dependence on radial position $r$ as $\lambda_{0} r^{\beta}$ varies as (Parker 1963; see Eq. C1 in Ng et al. (2003)

$$
n_{x}(r, t)=\frac{1}{4 \pi \Gamma(\varepsilon)}\left(\frac{\varepsilon}{3}\right)^{2 \varepsilon-1}\left(\frac{3}{\lambda_{0} v t}\right)^{\varepsilon} \exp \left[\frac{-3 r^{(2-\beta)}}{(2-\beta)^{2} \lambda_{0} v t}\right]
$$

where $\varepsilon=3 /(2-\beta)$ and $\beta$ must be less than 2 .

If we examine the ratio of species $\mathrm{X}$ and $\mathrm{Y}$, where $\lambda$ is a power of rigidity $P$ and where $L=\lambda_{X} / \lambda_{Y}=R^{\alpha}=\left(\left(A_{\mathrm{X}} / Q_{\mathrm{X}}\right) /\left(A_{\mathrm{Y}} / Q_{\mathrm{Y}}\right)\right)^{\alpha}$, as a result of the rigidity dependence of $\lambda$, and $\tau=3 r^{2-\beta} /\left[\lambda_{Y}(2-\beta)^{2} v\right]$, remembering that $\log x \approx 1-1 / x$, it can easily be shown (e.g. Reames 2016a, b) that

$$
\mathrm{X} / \mathrm{Y}=L^{-\varepsilon} \exp [(1-1 / L) \tau / t] \approx L^{\tau / t-\varepsilon}
$$

The ratio in Eq. (5.2) is the enhancement or suppression relative to that ratio at the SEP source and does not include any pre-enhanced impulsive suprathermal ions, although those are also power-law in form. Thus, relative abundances vary approximately as a power of $A / Q$. This will prove to be important in determining sourceplasma temperatures (Sect. 5.6). If the ratio $R>1$, as for $\mathrm{Fe} / \mathrm{O}$, the abundance ratio, $\mathrm{X} / \mathrm{Y}$ begins at infinity and falls asymptotically to $R^{-\alpha \varepsilon}$. Ratios begin at infinity because diffusion does not account for the particle transit time at the onset. Breneman and Stone (1985) observed that element abundance enhancements were power laws in $A / Q$, rising with $A / Q$ in some SEP events and falling in others as we saw in Fig. 2.5 in Sect. 2.5.1. In standard diffusion theory, scattering does not change with time; thus, the waves affect the particles, but the particles have no affect on the waves (defying energy conservation).

\subsubsection{Wave Growth}

The amplification of Alfvén waves by streaming protons has been discussed in textbooks on plasma physics for many years (e.g. Stix 1962, 1992; Melrose 1980; see also $\mathrm{Ng}$ et al. 2003; Rice et al. 2003; Li et al. 2005). In quasi-linear theory, ions, streaming along the magnetic field $\boldsymbol{B}$, resonate with Alfvén waves of wave number $k$ :

$$
k \approx \frac{B}{\mu P}
$$

in the rest frame of the waves. Here $P=p c / Q e$ is the rigidity of a particle of charge $Q e$, and momentum $p$, and $\mu$ is the cosine of its pitch angle relative to $\boldsymbol{B}$. 
Equation (5.3) results from quasi-linear theory (QLT) where particles are assumed to orbit the unperturbed field and the electric field vector of the resonant circularly-polarized Alfvén wave rotates so as to maintain its phase relative to the direction of rotation of the gyrating particle. This resonance maximizes the transfer of energy between the wave and the particle, seen as pitch-angle scattering in the rest frame of the wave, or wave frame, approximately the plasma rest frame.

The growth rate of the $\sigma$ polarization mode of Alfvén waves (see $\mathrm{Ng}$ et al. 2003; Stix 1992; Melrose 1980) produced by protons is clearest and simplest in the wave frame, where it is given by

$$
\gamma_{\sigma}(k)=2 \pi^{2} g_{\sigma} e^{3} c V_{A} \iint d \mu d P \frac{P^{3}}{W^{2}} R_{\mu \mu}^{\sigma} \frac{\partial f_{H}^{+}}{\partial \mu}
$$

where $g_{\sigma}= \pm 1$ for outward (inward) wave direction and $f_{\mathrm{H}}{ }^{ \pm}$is the proton phasespace density in each corresponding wave frame. Here $W$ is the total proton energy, and $R_{\mu \mu}^{\sigma}$ is the resonance function (see $\mathrm{Ng}$ and Reames 1995; $\mathrm{Ng}$ et al. 2003) that imposes the resonance condition (Eq. 5.3) while allowing for resonance broadening near $\mu \approx 0$. Resonance broadening overcomes the limitation of QLT that prevents scattering across $\mu=0$ ( $\mathrm{Ng}$ and Reames 1995). If we can ignore the effects of slow propagation of the waves, then the wave intensity of the $\sigma$ mode, $I_{\sigma}(k, r, t)$ obeys the simple equation

$$
\frac{\partial I_{\sigma}(k, r, t)}{\partial t}=\gamma_{\sigma}(k, r, t) I_{\sigma}(k, r, t)
$$

also in the wave frame, where we have explicitly shown the dependence upon space $r$ and time $t$, which may be quite significant. We will see that the pitch-angle diffusion coefficient for protons depends linearly upon the intensity of resonant waves (Sect. 5.1.3). Equation (5.5) was used by Ng and Reames (1994) to study time-dependent wave growth during proton transport that was quantitatively consistent with the streaming limit as we will see in Sect. 5.1.5.

Thus, streaming protons grow the waves, and those waves scatter the subsequent protons to reduce the streaming and the wave growth. This causes the scattering mean free paths to vary in both time and space $(\mathrm{Ng}$ et al. 1999, 2003). While the wave growth caused by heavier ions is negligible, they respond to the waves in ways that are not always obvious, a priori. Waves, grown by protons at a particular value of $\mu P$, resonate with other energies and other species with the same value of $\mu P$, as shown in Eq. (5.3).

Wave growth is commonly combined with quasi-parallel shock acceleration, where scattering is especially important. However, wave growth is entirely a transport phenomenon, its dependence upon the particles is only through $\partial f_{\mathrm{H}}{ }^{ \pm} / \partial \mu$; it is otherwise completely independent of the nature of the proton source. Wave growth will also be important near quasi-perpendicular shocks when streaming intensities of protons become large. This point is sometimes overlooked by students. 
Working in the wave frame is illustrative but inconvenient when both inward and outward waves are present and when the Alfvén speed $V_{\mathrm{A}}$ decreases as $r^{-1}$ with distance. Transforming to the plasma frame introduces terms of order $\left(V_{S W}+g_{\sigma} V_{\mathrm{A}}\right) / v$ (see e.g. Ng et al. 2003).

\subsubsection{Particle Transport}

The equation of particle transport may be simplified in the fixed inertial frame where $f$ is the phase-space density of a given particle species averaged over gyrophase (Roelof 1969; Ng and Reames 1994; Ng et al. 1999).

$$
\frac{\partial f}{\partial t}+\mu \nu \frac{\partial f}{\partial r}+\frac{1-\mu^{2}}{r} \nu \frac{\partial f}{\partial \mu}-\frac{\partial}{\partial \mu}\left(D_{\mu \mu} \frac{\partial f}{\partial \mu}\right)=G
$$

The third term in Eq. (5.6) represents focusing of the particles in the diverging magnetic field while the fourth term represents pitch-angle scattering with the diffusion coefficient $D_{\mu \mu}$. Here $v$ is the particle speed, $\mu$ is its pitch angle cosine, and the term $G$ on the right-hand side of the equation represents particle sources, for example, it might be a power-law energy spectrum times a delta-function at the radial location of a shock wave.

The diffusion coefficient $D_{\mu \mu}$ is given by

$$
D_{\mu \mu}=\frac{v^{2}}{4 P^{2}} \sum_{\sigma} \int d k I_{\sigma} R_{\mu \mu}^{\sigma}
$$

where $P$ is the particle rigidity and $\sigma$ runs over wave modes. The wave intensity $I_{\sigma}$ and the resonance function $R_{\mu \mu}^{\sigma}$ were discussed in the previous section.

The set of Eqs. (5.4-5.7) completely describe the evolution of both particles and waves and their coupling. Equation (5.4) shows that the growth of waves is controlled by the streaming particles and Eq. (5.7) relates the particle scattering to the intensity of waves. Scattering causes wave growth as a direct consequence of energy conservation (Ng et al. 2003, Appendix B).

\subsubsection{Initial Abundance Ratios}

We noted above that in diffusion theory, when $\lambda$ has a power-law dependence on rigidity, hence upon $A / Q$, ratios like $\mathrm{Fe} / \mathrm{O}$ or $\mathrm{He} / \mathrm{H}$ begin with large enhancements that decrease with time. While this occurs for small gradual SEP events, Fig. 5.2 shows that $\mathrm{He} / \mathrm{H}$ can reverse in large SEP events where wave growth becomes important. This is an example of a case where waves that control the arrival of protons depend upon the proton's velocity, but those that affect He, for example, depends upon protons of a much higher velocity, at their common value of $\mu P$; those protons already arrived and generated their waves much earlier. 


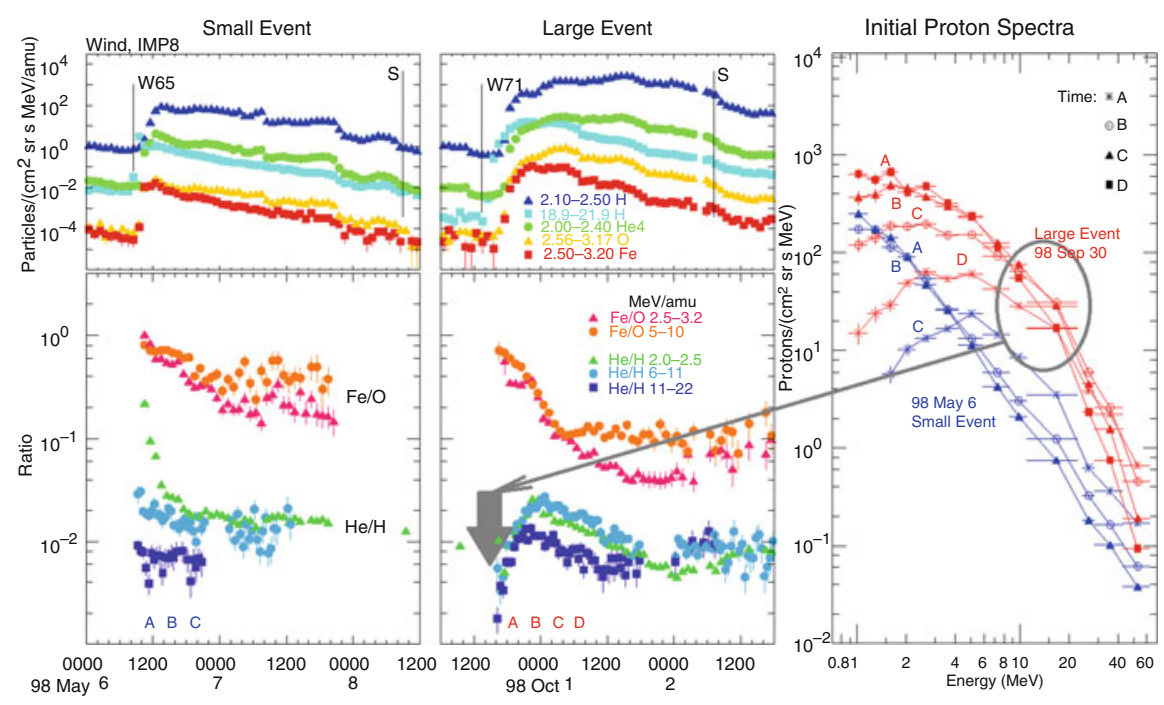

Fig. 5.2 Particle intensities and abundance ratios are shown for small (left) and large (center) gradual SEP events (Reames et al. 2000 (C) AAS). Initially He/H ratios fall in the small event but rise in the large one. Proton spectra at times A, B, C, and D, (shown in the right panel) are much more intense ( gray circle) in the large October event ( red), their greater wave generation causing initial suppression of $\mathrm{He} / \mathrm{H}$ (gray arrow) since $\mathrm{He}$ in the ratio resonates with waves produced by copious higher-energy protons that arrived much earlier than the $\mathrm{H}$ in the ratio

Why does the initial behavior reverse for $\mathrm{He} / \mathrm{H}$ in the large event? The early ions stream out into space from the event with $\mu \approx 1$ with few resonant Alfvén waves and little scattering. The $\mathrm{H}$ at $2 \mathrm{MeV}$, for example, has suffered little scattering and is only beginning to make its own resonant waves. He at $2 \mathrm{MeV} \mathrm{amu}^{-1}$, however, is scattered by waves that were amplified by $8-\mathrm{MeV}$ protons (same rigidity) that came out much earlier. If the intensity of $8-\mathrm{MeV}$ protons is high (i.e. a big event), they arrive earlier and generate waves so the newly arriving $2-\mathrm{MeV} \mathrm{amu}^{-1} \mathrm{He}$ will be trapped near the shock since it is scattered much more than the 2-MeV H. Similar logic applies to $\mathrm{He} / \mathrm{H}$ at higher energies. This effect does not occur for $\mathrm{Fe} / \mathrm{O}$ since both species are scattered by earlier proton-generated waves. Waves that scatter Fe are coupled to protons of quite high energy, which are less intense, so they actually increase $\mathrm{Fe} / \mathrm{O}$ initially. The progression of enhancements is modeled by $\mathrm{Ng}$ et al. (2003).

\subsubsection{The Streaming Limit}

In a study of large SEP events observed at the Helios spacecraft in solar orbit, Reames (1990) noticed that there was an early plateau period (see Fig. 5.1) during large SEP events near $1 \mathrm{AU}$, where the proton intensities seemed to have an upper limit of intensity as shown in Fig. 5.3. 
Fig. 5.3 Initial intensities of 3-6 MeV protons are shown overlapped for six large SEP events, all near $1 \mathrm{AU}$. Intensities do not seem to exceed $\sim 200\left(\mathrm{~cm}^{2} \mathrm{sr}\right.$ $\mathrm{s} \mathrm{MeV})^{-1}$ early in the events, but can become much higher later when shock peaks arrive (Reames 1990 (C) AAS)

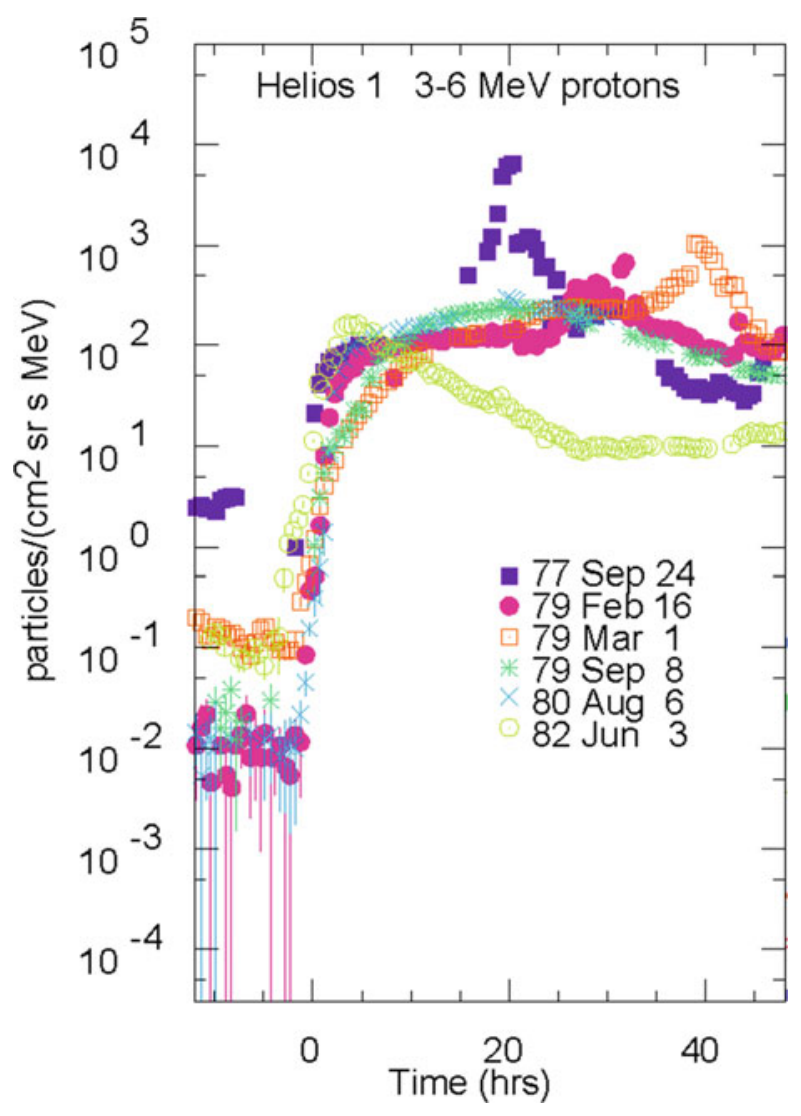

The intensities can rise much higher at the shock peaks, which are at the particle source, because particles at the shock have no net streaming. The streaming limit is a transport phenomenon.

Imagine an experiment that slowly increases the SEP injection intensity at a source near the Sun. At first, the intensity at 1 AU would increase proportionally. Then, at higher source intensities, wave growth would begin to scatter and trap the particles, with most wave growth near the source where intensities are highest. Eventually, further increasing flow from the source would increase the wave growth and scattering so much that the intensity at $1 \mathrm{AU}$ would no longer increase. This is the "streaming limit" that also emerges from theoretical transport models that include wave growth (e.g. Lee 1983, 2005; Ng and Reames 1994; Ng et al. 1999, 2003, 2012). The intensity behavior at 1 AU vs. that at the source near the Sun is shown in the left panel of Fig. 5.4 while the right panel shows the spatial dependence caused by increasing injection levels at the source.

Note that the wave growth depends upon the absolute value of the streaming intensity and the parameters shown in Eq. (5.4); there are no arbitrarily adjustable 

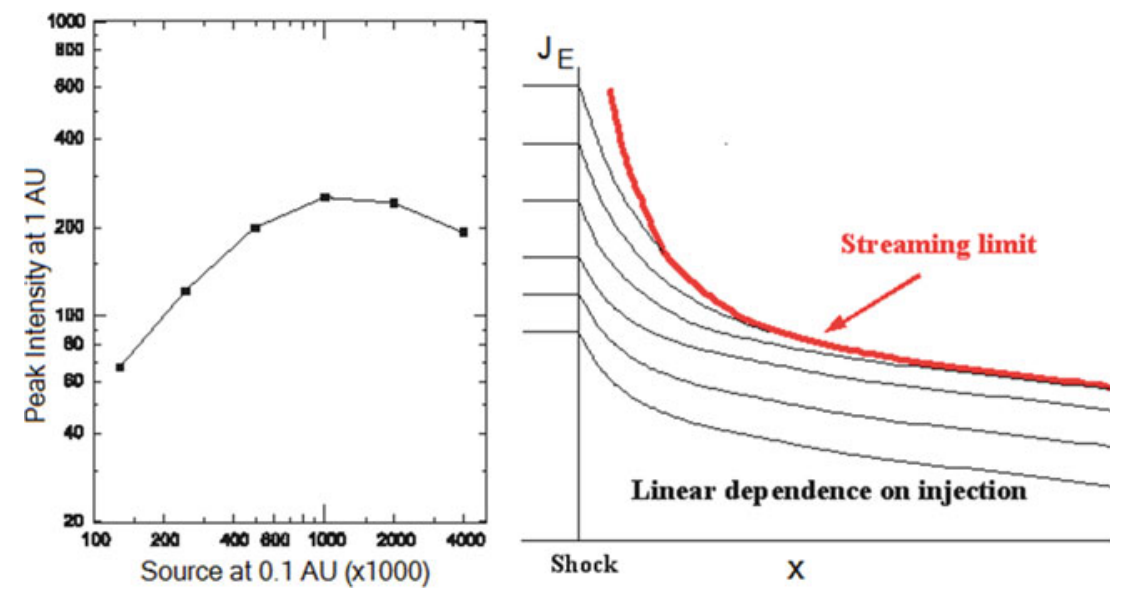

Fig. 5.4 The left panel shows intensity at $1 \mathrm{AU}$ vs. that at $0.1 \mathrm{AU}$. The right panel shows the spatial variation as the source intensity level is increased with linear behavior at low intensities (see $\mathrm{Ng}$ and Reames 1994; Ng et al. 2003, 2012)

parameters. The peak intensity in the left panel of Fig. 5.4 is just over $200\left(\mathrm{~cm}^{2} \mathrm{sr}\right.$ $\mathrm{s} \mathrm{MeV})^{-1}$, similar to the value observed in Fig. 5.3.

However, the plateau intensities in the largest gradual SEP events can involve more than just waves that are self-generated by particles of a single energy. They can involve waves generated by higher-energy protons that contribute to the scattering of lower-energy ions by coupling through the $\mu$ dependence of Eq. (5.3). These waves preferentially retard the lower-energy particles and flatten the power-law source spectra on the plateau as seen in the left panel of Fig. 5.5. Intense protons of 10-100 MeV stream out early, generating waves as they scatter toward smaller $\mu$. Waves generated at high $P$ and low $\mu$ resonate with ions of low $P$ and $\mu \approx 1$ which are coming behind more slowly. Thus, waves amplified by protons of $10 \mathrm{MeV}$ at $\mu \approx 0.5$ will scatter protons at $2.5 \mathrm{MeV}$ and $\mu \approx 1$, retarding their flow and thus flattening their spectrum at 1 AU.

Some proof of this mechanism in given by its absence in the 2 May 1998 SEP event; its plateau proton spectrum is shown in the right panel of Fig. 5.5. The spectrum in this event remains a power law since the intensity of $10 \mathrm{MeV}$ protons is two orders of magnitude smaller than that in 28 October 2003. The low intensities of 10-100 MeV protons do not generate enough waves to suppress the low-energy spectrum in the May event. The theoretical fits to these spectra, shown in Fig. 5.5, support this explanation. Wave growth can control spectral shape. 

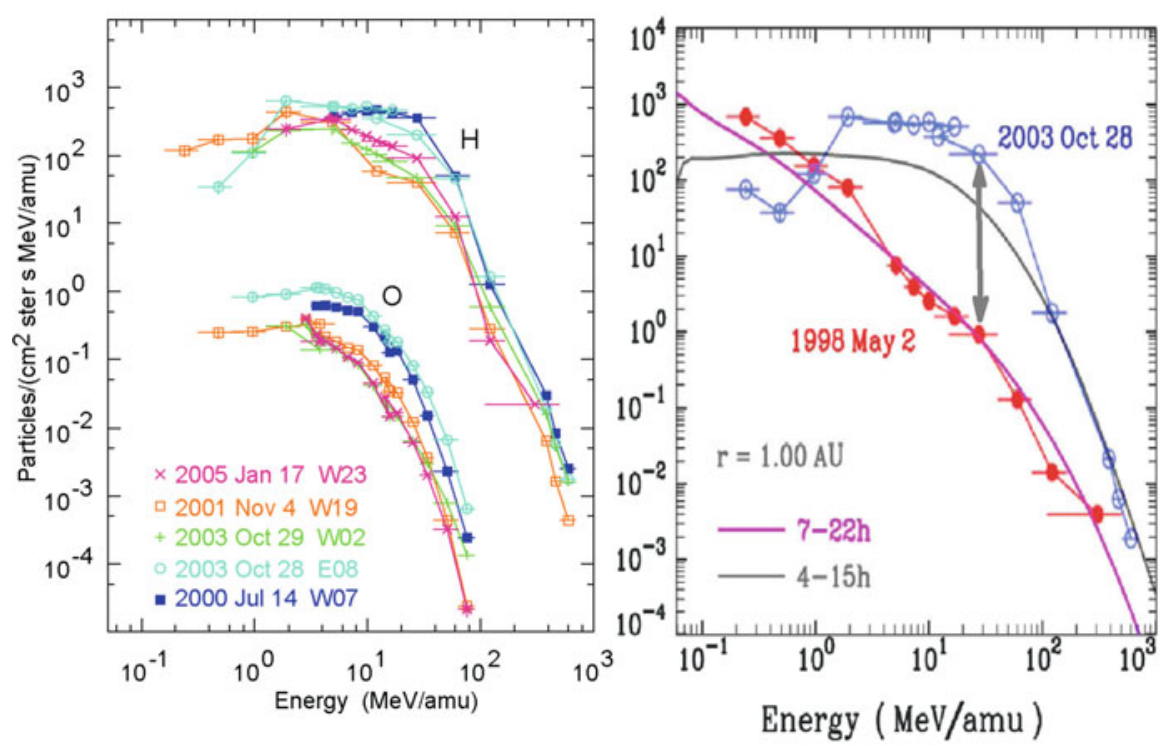

Fig. 5.5 The left panel shows energy spectra of $\mathrm{H}$ and $\mathrm{O}$ in five large gradual SEP events (all GLEs) that are flattened at low energies (Reames and Ng 2010 (C) AAS). The right panel shows that the small event of 2 May 1998, with greatly reduced $\mathrm{H}$ intensities at 10-100 MeV (gray arrows), cannot generate enough waves to suppress lower energies. Model fits to the spectra are shown in gray and purple curves (see $\mathrm{Ng}$ et al. 2012)

\subsubsection{Electron Transport}

Non-relativistic electrons cannot resonate with Alfvén waves, so they do not participate in much of the physics we have just described. Low-energy electrons usually propagate scatter free with highly-anisotropic angular distributions mainly because of absorption by the solar wind of $0.1-1 \mathrm{~Hz}$ frequencies that would resonate with these electrons. Electron spectra often show a break in the $\sim 100-\mathrm{keV}$ energy region. Above the break the spectrum steepens and the width of the angular distribution broadens as scattering becomes much more important (see Tan et al. 2011). It is sometimes erroneously concluded that $1 \mathrm{MeV}$ electrons are accelerated much later than those at 20-50 keV in SEP events; this apparent delay could result from transport rather than acceleration (Strauss and le Roux 2019).

\subsection{Angular Distributions}

Not surprisingly, angular distributions also show the effects of increased scattering when high proton intensities amplify waves. This is seen in the angular distributions of $\mathrm{H}$ and $\mathrm{He}$ ions in large and small SEP events as shown in Fig. 5.6. The particle intensities remain clustered along the field direction around $180^{\circ}$ for more than a day 


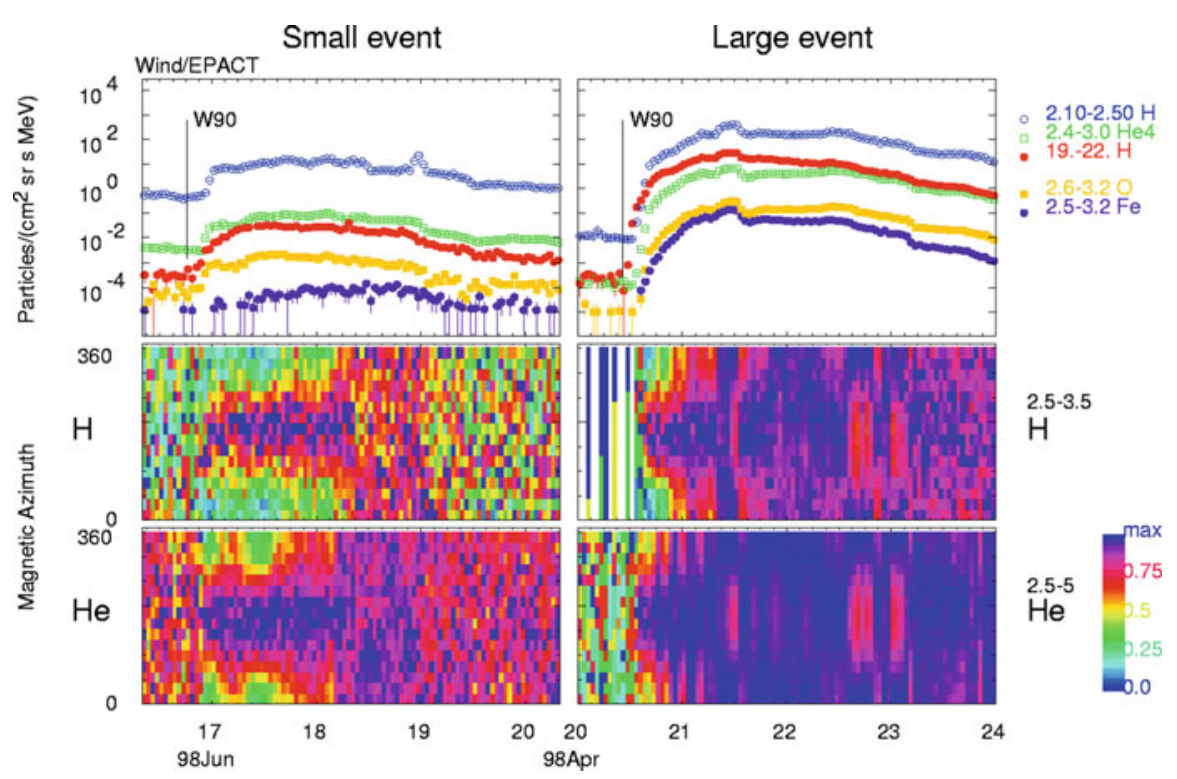

Fig. 5.6 Intensities (top) and angular distributions, relative to $\boldsymbol{B}$, for $\mathrm{H}$ (middle) and ${ }^{4} \mathrm{He}$ (bottom) are shown for small (left) and large (right) gradual SEP events. Note the much higher intensity of the (red) 19-22 MeV protons in the upper right panel

in the angular distributions of the small event on the left in Fig. 5.6 but, in the more intense event on the right, the angular distributions begin to spread in only a few hours.

Of course, the scattering and the wave growth depend upon the initial wave intensity. However, small impulsive and gradual events usually remain scatter-free and angular distributions rapidly isotropize in more-intense gradual events and especially in GLEs (see Reames et al. 2001). Most SEP events begin nearly scatter free at energies above a few $\mathrm{MeV} \mathrm{amu}^{-1}$, but not at low energies where $\mu$-coupling shown in Fig. 5.5 applies and traps ions with energies below a few $\mathrm{MeV}$ amu ${ }^{-1}$ near their source.

\subsection{Models and Shock Acceleration}

General information about shock formation and acceleration may be found in comprehensive review articles (Jones and Ellison 1991; Lee et al. 2012; Desai and Giacalone 2016). However, there is such compelling experimental evidence of wave growth in the larger gradual SEP events that we focus on models that include it.

The earliest time-equilibrium model of shock acceleration with self-consistent treatment of particles and waves was the work of Bell (1978a, b) on GCRs, which was subsequently adapted to interplanetary shocks by Lee (1983). Shock models were applied to acceleration of $\mathrm{GeV}$ protons in the corona by Zank et al. (2000, see 

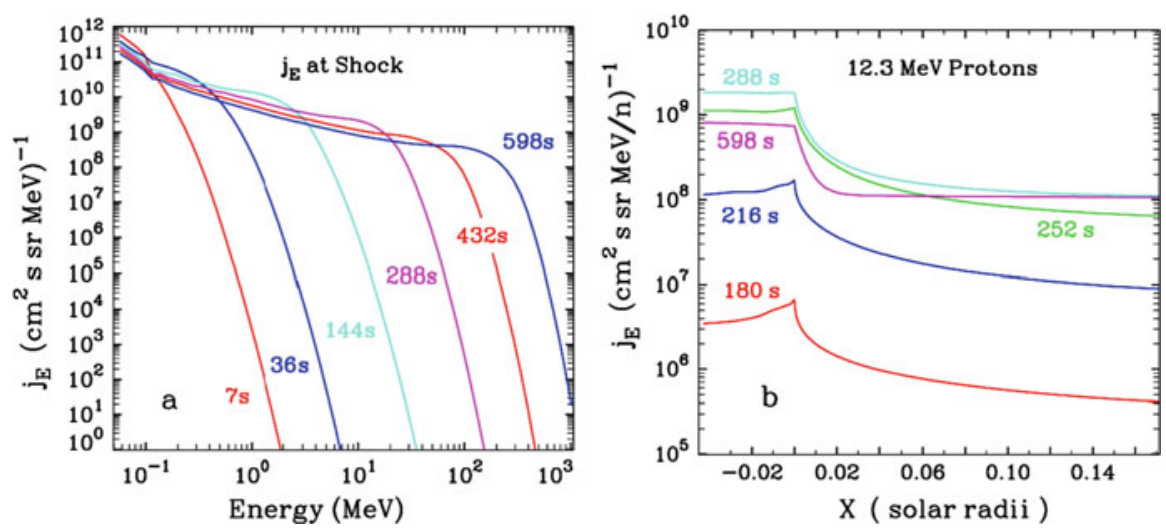

Fig. 5.7 The left panel shows the time evolution of the proton energy spectrum at the shock for the first $\sim 10 \mathrm{~min}$. The right panel shows the time evolution of the spatial distribution of $12.3 \mathrm{MeV}$ protons upstream of the shock. Once accelerated at $\sim 3 \mathrm{~min}, 12.3 \mathrm{MeV}$ proton intensities form a streaming limit within $0.1 \mathrm{R}_{\mathrm{S}}$ of the shock at $\sim 4.2 \mathrm{~min}$ ( $\mathrm{Ng}$ and Reames 2008 (C) AAS)

also Lee 2005, Sandroos and Vainio 2007, Zank et al. 2007, Afanasiev et al. 2016, 2018).

The time-dependent self-consistent model of particle transport with wave amplification ( $\mathrm{Ng}$ et al. 2003) was applied to shock acceleration by $\mathrm{Ng}$ and Reames (2008) resulting in modeling of the time-evolution of the proton spectra at the shock shown in Fig. 5.7 along with the evolution of the radial dependence of the intensity upstream of the shock for a given energy proton. A streaming limit soon forms within $0.1 R_{S}$ of the shock as seen in the right panel.

An interesting feature of the time-dependent numerical acceleration calculations is the growth of waves as the proton spectrum grows to higher energy. With the growth of waves that resonate with particles of the highest energy $E_{1}$ and rigidity $P_{1}$, some protons will begin to be accelerated to still-higher energy $E_{2}$ and rigidity $P_{2}$. Initially, the only waves that can trap ions at $E_{2}$ are those that resonate with protons with $\mu_{2}<P_{1} / P_{2}$, i.e. only at small $\mu_{2}$ can ions at the new energy find resonant waves generated by lower-energy protons. Thus at each new energy the particles begin with a pancake distribution at small $\mu$ ( $\mathrm{Ng}$ and Reames 2008).

The Ng and Reames (2008) model prevents the scattering from approaching the Bohm limit by requiring that the scattering mean free path be more than three times the particle gyroradius, so that the quasi-linear approximation remains valid. This makes the maximum energy lower and the acceleration rate slower than that in the calculation of Zank et al. (2000), who assumed the more-likely Bohm limit where scattering mean free path equals the particle gyroradius, i.e. $\delta B / B \approx 1$, as has been observed in strong shocks (Lario and Decker 2002; Terasawa et al. 2006). It is also true that an oblique shock, where ions gain energy in the $\boldsymbol{V}_{\boldsymbol{S}} \times \boldsymbol{B}$ electric field, can affect the acceleration time and maximum energy by increasing the particle energy gained on each traversal of the shock. We would speculate that a fast shock 
traversing a sufficiently dense seed population should have no trouble accelerating $\mathrm{GeV}$ protons in a minute or so.

$\mathrm{Ng}$ and Reames (2008) solved the self-consistent time-dependent wave-particle interaction while Zank et al. (2000) used a series of instantaneous steady-state solutions in their PATH (Particle Acceleration and Transport in the Heliosphere) model. The latter simplicity gave a better determination of maximum particle energies of $\mathrm{GeV}$ and allowed the inclusion of more-realistic two-dimensional versions of the CME and shock in the PATH models, including oblique shocks (Li et al. 2012) and perpendicular diffusion of the particles caused by random walk of the magnetic fields (Hu et al. 2017, 2018).

It can not escape our attention that it is much easier for theoreticians to work in a universe where particle scattering is constant in time, and waves never grow. Quasiperpendicular shocks need no change in scattering to increase acceleration, only a small change in $\theta_{\mathrm{Bn}}$. Such approximations are often useful in making tractable solutions to explore specific functional dependences. However, observations show that wave growth dominates the largest SEP events. Further realistic studies that include it could help advance our understanding of these important events.

\subsection{Shock Acceleration In Situ}

Traveling interplanetary shock waves near Earth are the local continuation of the CME-driven shock waves that produce gradual SEP events. These shocks provide an opportunity to directly measure, in situ, the properties of accelerated particles together with the characteristics of the shock and its driver under an extremely wide variety of shock conditions (see e.g. Berdichevsky et al. 2000). Desai et al. (2003) showed that low-energy ion abundances near the shock peak were much more closely related to ambient abundances of those ions upstream of the shock than to the abundances of the corresponding elements in the solar wind, as might be expected from our discussion of the seed population in Sect. 2.5.3. Desai et al. (2004) found that energy spectra at the shocks were better correlated with the spectra upstream than with those expected from the shock compression ratio. Especially for low-energy ions, shock acceleration persists far out from the Sun and tends to reaccelerate ions from the same population that was accelerated earlier.

The choice of a location to measure the ambient, background, or reference abundances and spectra upstream of the shock is difficult. If it is chosen prior to the time that shock leaves the Sun, perhaps $~ 2-3$ days before the shock arrival, then solar rotation insures that background is sampled at a longitude of 26-40 degrees to the west of the longitude sampled at the shock peak. If it is chosen hours prior to the shock arrival, background will be dominated by particles accelerated earlier by the same shock. Neither choice is ideal.

In effect, the re-acceleration of ions from the seed population found in the reservoir of an earlier event evokes the classical two-shock problem considered, for example, in the review by Axford (1981) and subsequently by Melrose and Pope 
(1993). Here, the integral equilibrium distribution function $f(p)$ of momentum $p$ of accelerated particles from a shock with compression ratio $s$ is

$$
f_{a}(p)=a p^{-a} \int_{0}^{p} d q q^{a-1} \phi(q)
$$

where $a=3 s /(s-1)$ and $\phi(p)$ is the injected distribution. If we take $\phi(p)$ as a delta function at $p_{0}$ we find a power-law spectrum $f_{\mathrm{a}}(p) \sim\left(p / p_{0}\right)^{-a}$ after the first shock. If we reapply Eq. (5.8), injecting $f_{\mathrm{a}}(p)$ into a shock with compression ratio $s$ ' and let $b=3 s^{\prime} /\left(s^{\prime}-1\right)$, we find that integrating the power law gives.

$$
f_{a, b}(p)=\frac{k a b}{p_{0}(b-a)}\left[\left(\frac{p}{p_{0}}\right)^{-a}-\left(\frac{p}{p_{0}}\right)^{-b}\right] \text { for } a \neq b .
$$

The corresponding intensity is $j(E)=p^{2} f(p)$.

Note that Eq. (5.9) is symmetric in the powers $a$ and $b$, and will be dominated by the shape of the hardest, flattest spectrum, either the background (i.e. $a$ ) or the new shock, $b$. Thus, it is no surprise that one finds local-shock spectra that are dominated by the shape of the upstream background spectrum (Desai et al. 2004; Reames 2012) produced earlier when the shock was stronger. It is quite often that ions with properties from an earlier epoch seem to be only "trapped" at a shock when their intensities are actually being increased by that shock. A further complication occurs when we include a spectral knee with a factor like exp. $\left(-E / E_{0}\right)$ (e.g. Ellison and Ramaty 1985; see also Mewaldt et al. 2012) to allow for the finite acceleration time. At energies above the knee, observers will find spectra that are much steeper than either the background or the expected equilibrium spectra.

These possibilities for spectral shapes were considered in the observations of Reames (2012), who studied ${ }^{4} \mathrm{He}$ spectra of $\sim 1-10 \mathrm{MeV}^{\mathrm{amu}}{ }^{-1}$ in 258 in situ interplanetary shocks observed near Earth by the Wind spacecraft (https://www. cfa.harvard.edu/shocks/). The purpose of this study was to determine which shock parameters were important to produce measurable particle acceleration and which were not. Figure 5.8 shows well-defined shock event No. 83.

Particle intensities in Fig. 5.8 are shown in the upper left panel, the plasma parameters: solar-wind speed $V_{\mathrm{SW}}$, magnetic field intensity $B$, and density $N$, in the lower left panel, and the shock and background spectra in the upper right panel. The times over which the two spectra are taken are shown in the upper left panel (Bk and Sh). This is a quasi-perpendicular shock with the angle between $B$ and the shock normal, $\theta_{\mathrm{Bn}}=80^{\circ} \pm 3^{\circ}$.

Figure 5.9 compares properties of the shocks in this study. The left panel shows a histogram of the shock speed distribution for all of the shocks and for the subset that showed measurable particle acceleration. High shock speed was the strongest determinant for measurable acceleration, followed by high shock compression ratio, and large $\theta_{\mathrm{Bn}}$. High background intensity was also important: more input produced more output. Measurable acceleration was more than twice as likely for shocks with $\theta_{\mathrm{Bn}}>60^{\circ}$ as for those with $\theta_{\mathrm{Bn}}<60^{\circ}$. Quasi-parallel shocks, i.e. small $\theta_{\mathrm{Bn}}$, may 


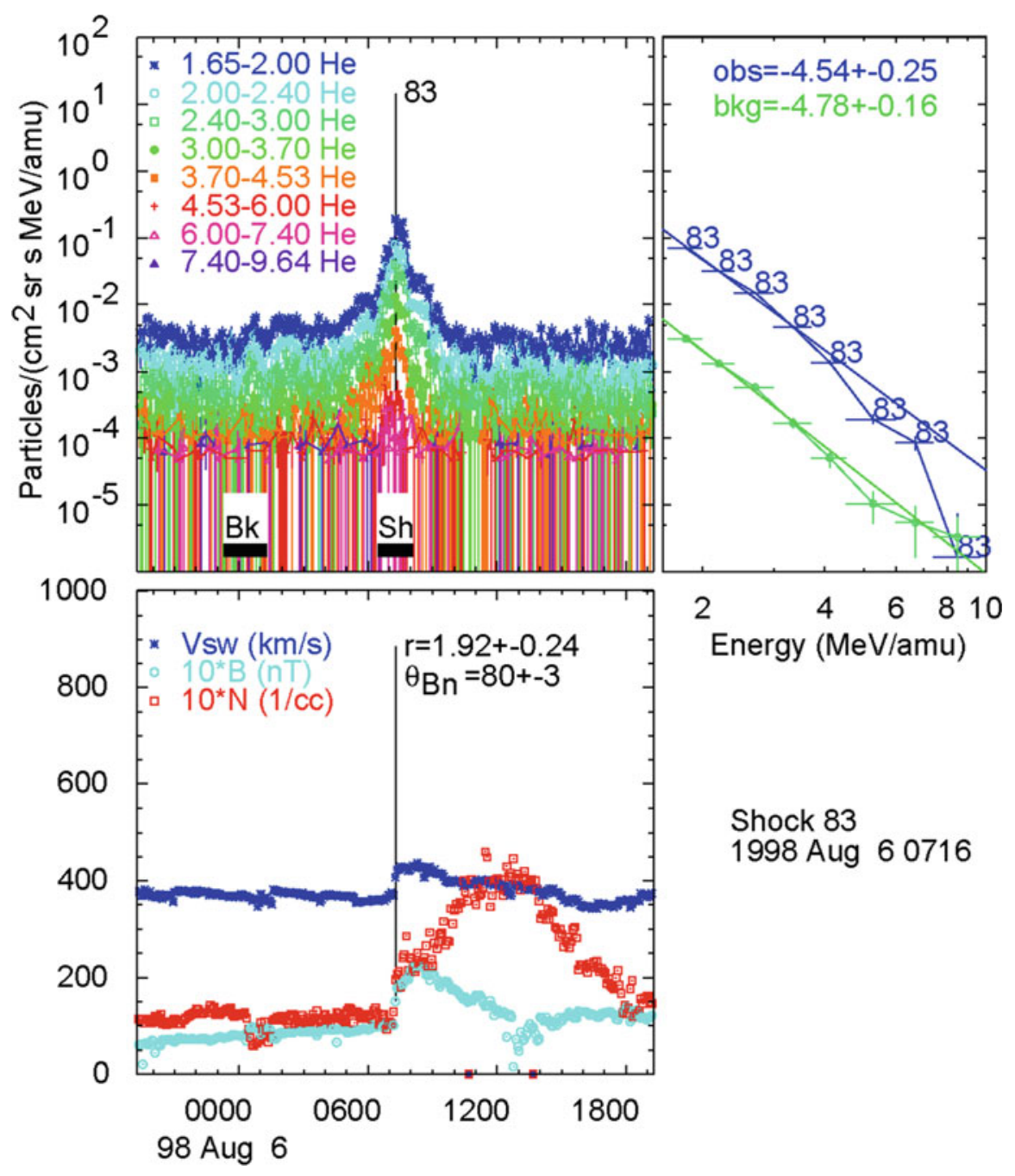

Fig. 5.8 Particle intensities are shown vs. time in the upper left panel with plasma parameters below for shock number 83 . Spectra of the shock and background are shown to the right with spectral slopes indicated (Reames 2012 (C) AAS)

have been more likely to have knee energies below the energy of observation. Recently, Zank et al. (2006) have suggested that "higher proton energies are achieved at quasi-parallel rather than highly perpendicular interplanetary shocks within 1 AU." The in situ observations (Reames 2012) show the opposite; quasiperpendicular shocks are favored; this difference likely occurs because ample pre-accelerated seed populations were available for these real shocks.

The right panel in Fig. 5.9 shows the background-corrected peak shock intensity of 1.6-2.0 MeV amu ${ }^{-1}{ }^{4} \mathrm{He}$ as a function of shock speed. The shock speed has a 


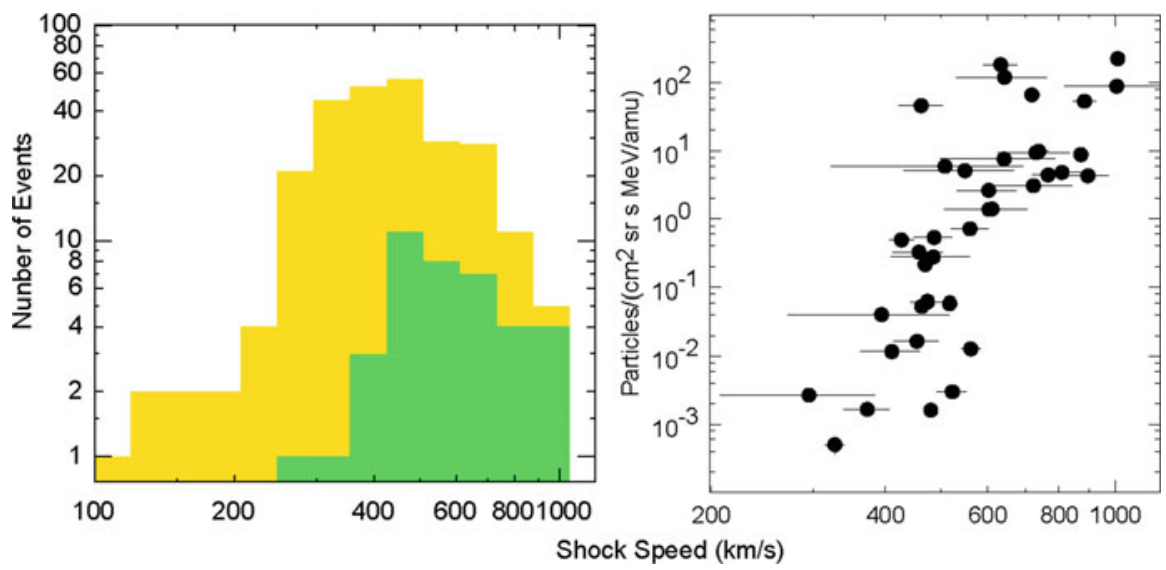

Fig. 5.9 The left panel shows the distribution of shock waves at 1 AU with measurable acceleration of $>1 \mathrm{MeV} \mathrm{amu}{ }^{-1}{ }^{4} \mathrm{He}$ vs. shock speed (green) within the distribution of all 258 shock waves vs. shock speed (yellow and green) observed by the Wind spacecraft. The right panel shows the background-corrected peak intensity of $1.6-2.0 \mathrm{MeV} \mathrm{amu}{ }^{-1}{ }^{4} \mathrm{He}$ versus shock speed for the shocks in situ. Shock speed is the strongest determinant of accelerated intensity for local shocks; this mirrors the correlated behavior of peak intensity versus CME speed in Fig. 2.12 (adapted from Reames 2012 (C) AAS, Reames 2013)

correlation coefficient of 0.80 with intensity. This correlation for in situ shocks mirrors the correlation of peak proton intensity with CME speed in Fig. 2.12 as modified by Rouillard et al. (2012) and shown in the lower right-hand panel of Fig. 3.4.

Particle intensities peak at the time of shock passage in nearly all of the events in the Reames (2012) study. However, sometimes intensities peak before or after shock passage when a spacecraft encounters magnetic flux tubes that connect it to a stronger part of the shock nearby, perhaps even one with a different value of $\theta_{\mathrm{Bn}}$.

Absolute intensities of accelerated particles are not directly predicted by acceleration theories that omit wave growth. The rate of injection of seed particles is treated as an adjustable parameter-more input results in more output, and this is the case for in situ events. However, streaming protons and increasing wave intensities can trap particles near the source. At a few powerful shock waves, such as 20 October 1989, it has been observed that the energy in energetic particles exceeds that in the plasma and magnetic field (Lario and Decker 2002). Those authors suggested that the peak intensities of particles up to $500 \mathrm{MeV}$ are simply trapped in a region of low density and low magnetic field near a shock. Maybe, but, how did they get there? Surely they were accelerated there near the peak intensity even though their spectrum may reflect shock properties from an earlier time (see Eq. 5.9). Perhaps the wave-trapped particles are in the process of destroying (i.e. pushing apart $B$ at) the shock that accelerated them. Another shock where the particle energy exceeds the magnetic energy is that of 6 November 2001, in Fig. 5.1 (C. K. Ng, private communication), where the sharp proton peak up to $700 \mathrm{MeV}$ shows a shock that 
is still clearly intact. This is the issue of "cosmic-ray-mediated" shocks discussed by Terasawa et al. (2006) for two additional interplanetary shocks. This is a fascinating process that can be observed, in situ, at some interplanetary shocks.

\subsection{Averaging SEP Abundances}

We began by discussing the reference abundances in Chap. 1 and comparing them with the solar photospheric abundances as a function of first ionization potential (FIP) in Fig. 1.6 (see also Chap. 8). The reference abundances are obtained by averaging over many gradual SEP events. Since the transport of particles varies as a power of $A / Q$ (see Eq. 5.2), different species such as $\mathrm{Fe}$ and $\mathrm{O}$ will be distributed differently in space and time, but these particles are likely to be conserved. If we can successfully average over time or space we will recover the source abundances. If this assumption is correct and our averaging is representative, the reference abundances will approach the coronal abundances. Evidence for the space-time distribution is shown in Fig. 5.10.

The SEP event on the East flank of the CME (W85 source, on the left in Fig. 5.10), shows enhancement of $\mathrm{Fe} / \mathrm{O}$ early then suppression later, since $\mathrm{Fe}$, with higher $A / Q$, scatters less than $\mathrm{O}$. Ne/O, involving similar values of $A / Q$, varies little. Solar rotation and the Parker spiral translates this time variation into a spatial one and the events toward the West flank of the CME show mainly depleted Fe/O.

\subsection{Source-Plasma Temperatures}

Since particle transport of gradual SEPs varies as a power of $A / Q$, and $Q$ varies with $T$, we can use this power law to find the source-plasma temperature $T$ that gives the best-fit pattern vs. $A / Q$, just as we did for impulsive events. Figure 5.11 (similar to Fig. 4.8) shows $A / Q$ vs. $T$ with $Q$ derived from the atomic physics.

The red shaded region in Fig. 5.11 is 2.5-3.2 MK, corresponding to active region temperatures that we found for the impulsive SEP events that reflect the temperature of active-region jets where the SEPs originate (see Sect. 4.6). As we decrease $T$ below this region, $\mathrm{O}$, then $\mathrm{N}$, then $\mathrm{C}$ move from the 0 -electron to the 2-electron closed shells. Meanwhile, $\mathrm{Ca}$, then $\mathrm{Ar}$, then $\mathrm{S}$, then $\mathrm{Si}$, then $\mathrm{Mg}$ move from the 2-electron to the 8-electron shells. Thus, we can tell the temperature by the pattern of abundance enhancements. We need only notice which elements are in which group; which elements have no enhancement like He; which elements are in the group with $\mathrm{Ne}$; which are in the group with Ar.

Figure 5.12 compares the observed pattern of enhancements early in a large gradual SEP event (on the left) scaled to the pattern of $A / Q$ (on the right). The patterns match best near $T \approx 0.6 \mathrm{MK}$, an unusually low temperature for an SEP event. Note that $\mathrm{C}, \mathrm{N}$, and $\mathrm{O}$ have moved well above $\mathrm{He}$ to the 2-electron shell with $\mathrm{Ne}$, while $\mathrm{Mg}, \mathrm{Si}$, and $\mathrm{S}$ have moved up to the 8-electron shell close to $\mathrm{Ar}$ and Ca. Patterns of enhancement in other SEP events are shown in Reames (2016a). 


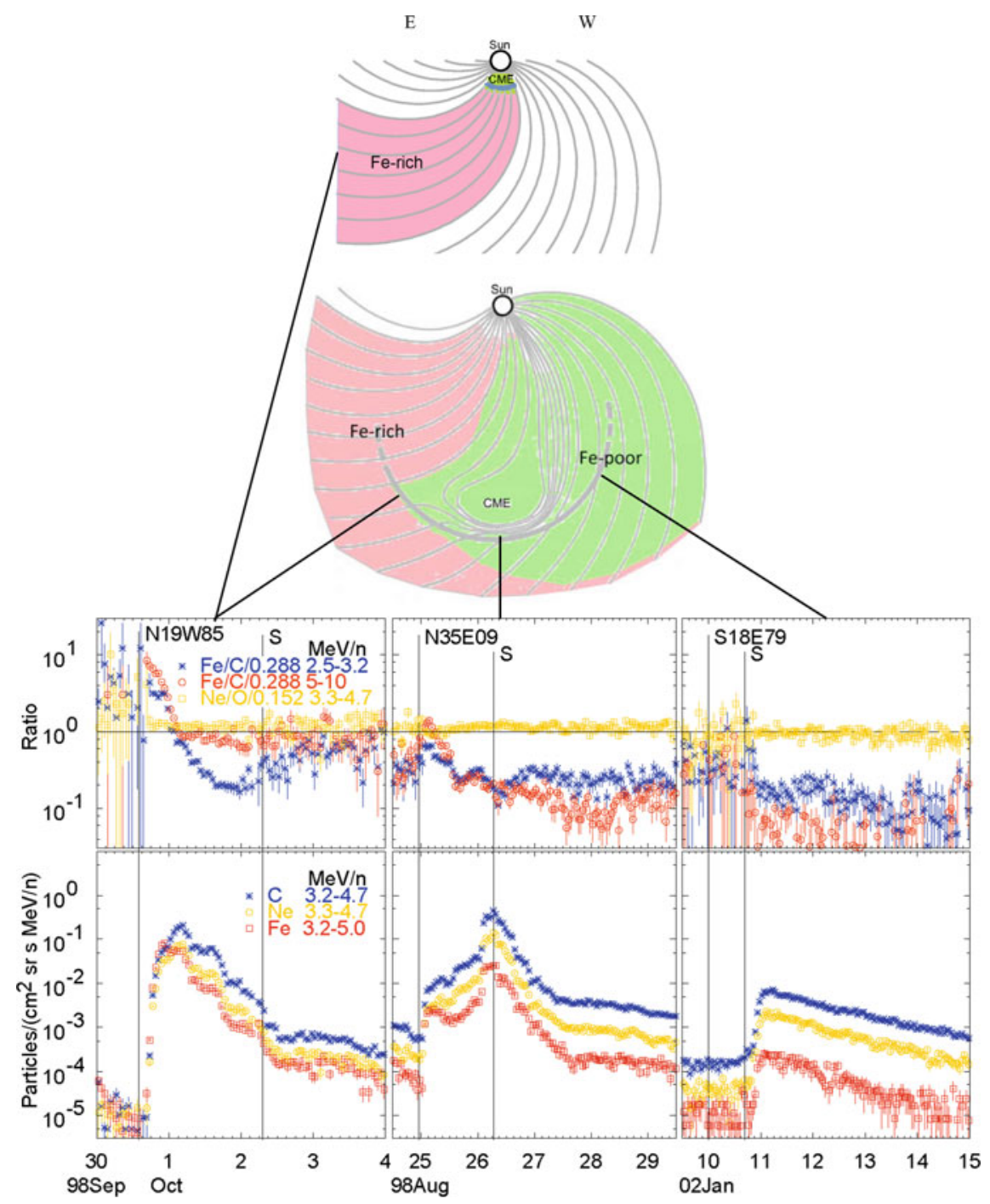

Fig. 5.10 Intensities of $\mathrm{C}, \mathrm{Ne}$, and $\mathrm{Fe}$ are shown for three gradual SEP events at different solar longitudes in the lower panels, relative abundances in the middle panels, and the location and evolution of a CME above (after Reames 2014 (C) Springer)

For the LEMT telescope on the Wind spacecraft, 8-h intervals during a large SEP event will provide adequate statistics for the rarer elements to determine enhancement patterns. For each 8-h period we can calculate least-squares fits of enhancement vs. $A / Q(T)$ for all values of $T$ in the range of interest and plot $\chi^{2}$ of the fit vs. $T$ (upper-right panel in Fig. 5.13). The minimum value of $\chi^{2}$ gives the best-fit temperature and power of $A / Q$ for that time interval. This process gives the 


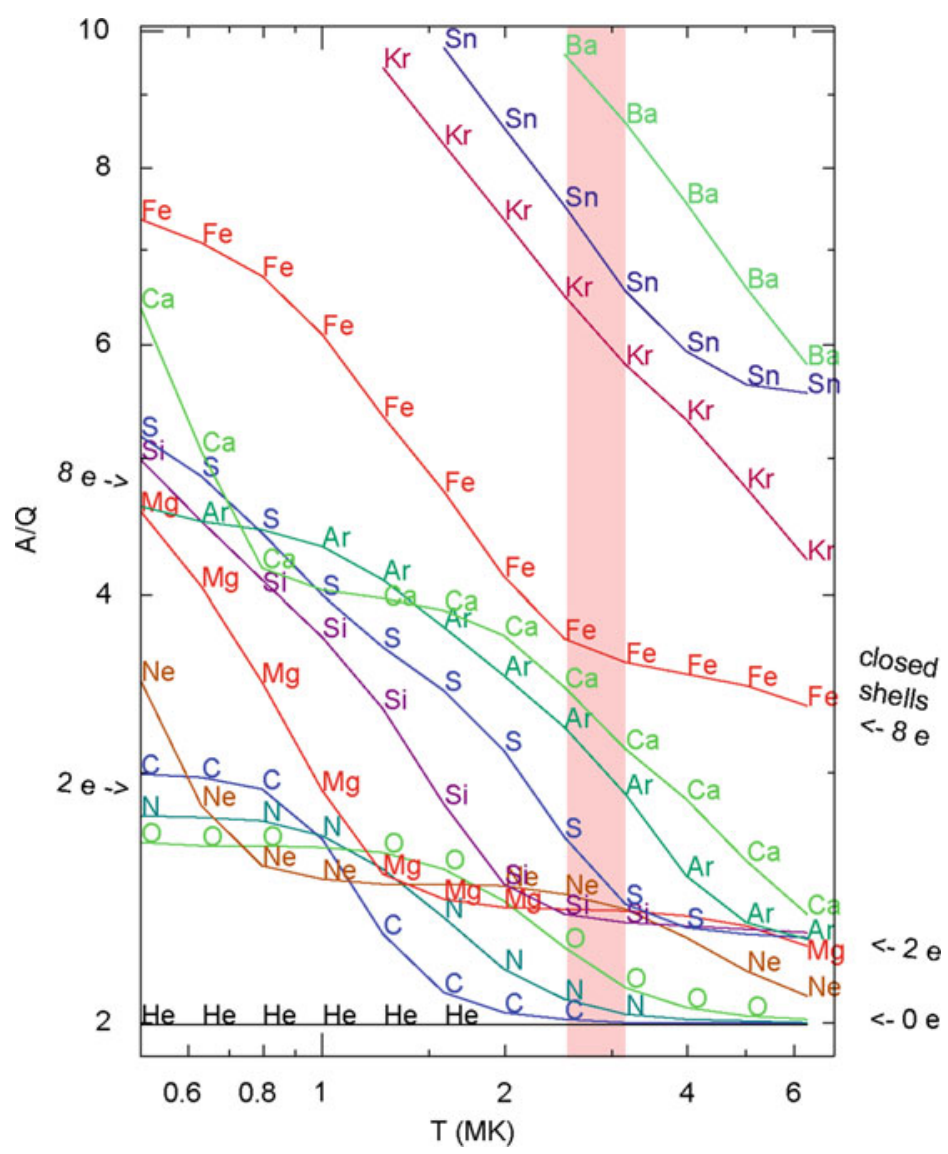

Fig. 5.11 $A / Q$ is plotted as a function of the theoretical equilibrium temperature for the elements named along each curve. Points are spaced 0.1 units of $\log _{10} T$ from 5.7 to 6.8 . Bands produced by closed electron shells with 0,2 , and 8 orbital electrons are indicated, He having no electrons at this $T$. Elements tend to move from one closed-shell group to another as the temperature changes. (Data for $Z \leq 28$ from Mazzotta et al. 1998, for $Z>28$ from Post et al. 1977)

source-plasma temperature as a function of time during an event, as shown in the upper-left panel of Fig. 5.13 for the event of 8 November 2000. For this event we find temperatures near $1 \mathrm{MK}$ for all time periods with either abundance enhancements or suppressions. For two of the time periods, the best fits to enhancement vs. $A / Q$ are shown in the lower-right panel of Fig. 5.13. However, for time periods when enhancements in the abundances are flat, neither enhanced nor suppressed relative to the coronal reference abundances, we cannot measure $T$, since any $A / Q$ values will fit and $\chi^{2}$ has no minimum. Larger enhancement or suppression of the abundances produces clearer minima in $\chi^{2}$ and smaller errors in $T$. 
2013 May 221600

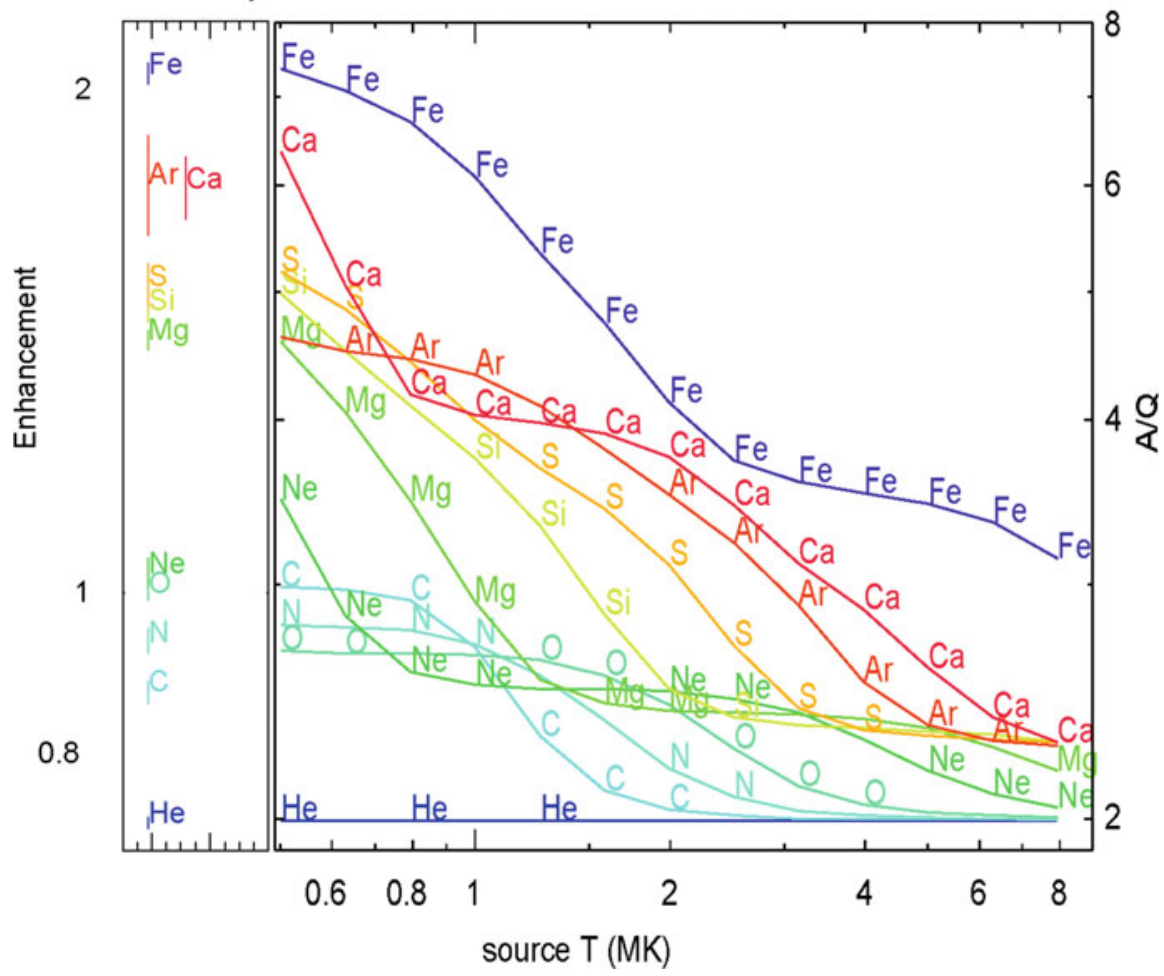

Fig. 5.12 The left panel shows the abundance enhancements at $\sim 3-5 \mathrm{MeV} \mathrm{amu}^{-1}$ observed early in the 22 May 2013 SEP event. The right panel compares a section of the $A / Q$ vs. $T$ plot from Fig. 5.11. The patterns match best at about 0.6 MK (Reames 2016a (C) Springer)

For 45 gradual SEP events that had reasonably well-defined temperatures, Reames (2016a) found:

- $69 \%$ (31 events) showed ambient coronal temperatures $T \leq 1.6 \mathrm{MK}$

- $24 \%$ (11 events) had $2.5 \leq T \leq 3.2 \mathrm{MK}$ active region temperatures, like impulsive SEP events where $T \approx 3 \mathrm{MK}$ is the typical temperature in solar jets associated with active regions (see Fig. 14 in Raouafi et al. 2016).

Some (11) of the events with ambient coronal temperatures showed a second minimum at the upper limit of $T$ in $\chi^{2}$ vs. $T$. These were originally attributed to the possible presence of stripped ions, but have been subsequently found to be artificially induced by variations in the source abundances of He (Reames 2017b; see Sect. 5.9). Subsequent studies omit $\mathrm{H}$ and He from fits (see Chap. 9).

While the gradual-event temperatures and fit parameters are not strongly correlated with any particular properties of the accelerating CME or shock, 

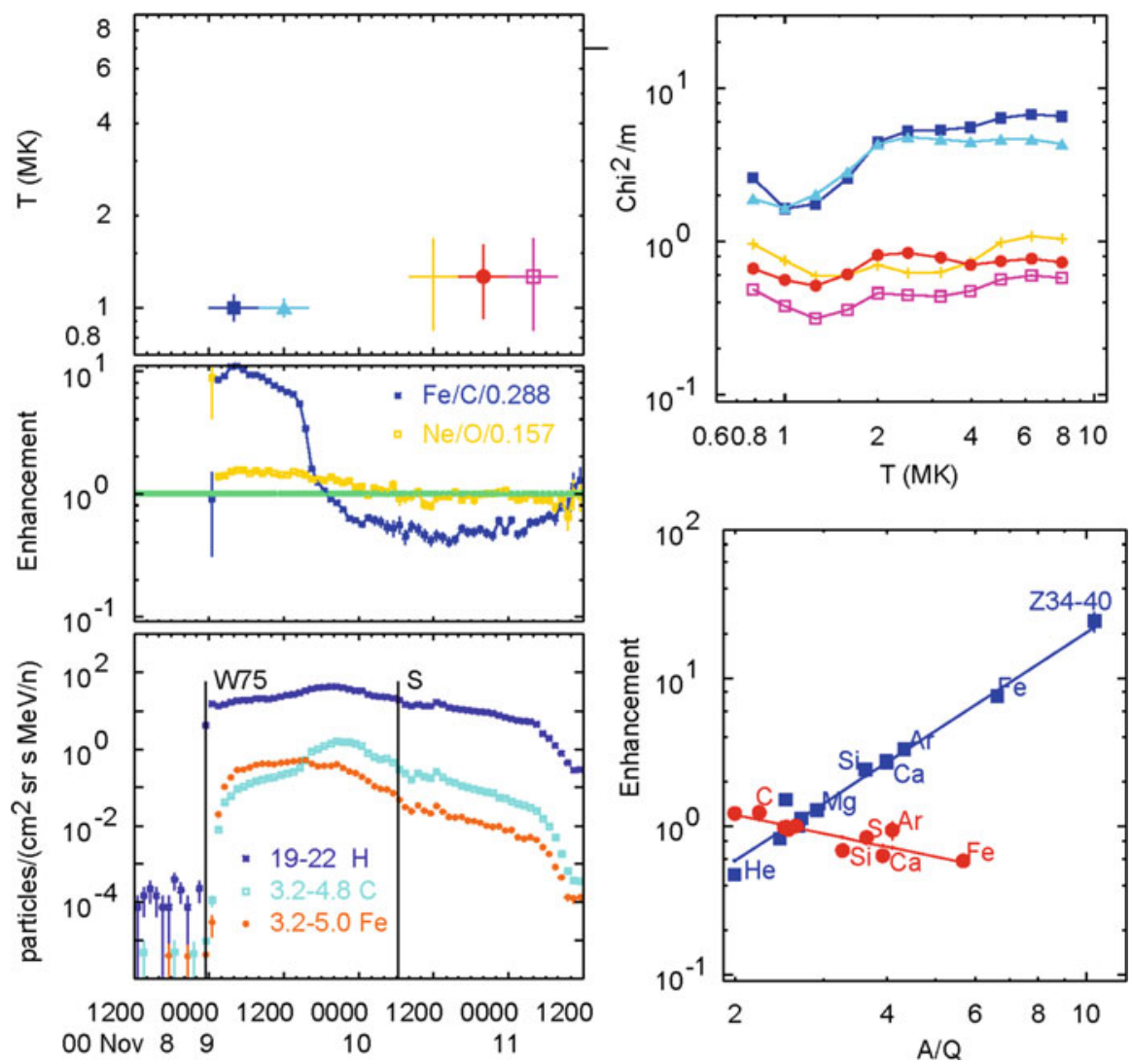

Fig. 5.13 Clockwise from the lower-left panel are the intensities of $\mathrm{H}, \mathrm{C}$, and $\mathrm{Fe}$ during the 8 November 2000 SEP event, the enhancements in $\mathrm{Fe}$ and $\mathrm{Ne}$ during the event, the best-fit temperatures in color-coded 8-hr intervals, values of $\chi^{2} / m$ vs. $T$ for each time interval (where $m$ is the number of degrees of freedom), and fits of enhancements from samples (blue filled square and red filled circle), early and late in the event, relative to O, vs. $A / Q$ (Reames 2016a (C) Springer)

Fig. 5.14 shows $T$ vs. CME speed. The un-weighted correlation coefficient is -0.49 for these events. Events that happen to be GLEs are identified in the figure; their temperature distribution and other properties are similar to those of the other gradual SEP events.

We now realize that attempts to study abundance cross-correlations in gradual SEP events were often ineffective because most variations were caused by temperature differences that previously were not known. For example, the average value of $\mathrm{Fe} / \mathrm{O}$ is a factor of $\sim 10$ higher in gradual events with $T=3.2 \mathrm{MK}$ than in those with $T=1.5 \mathrm{MK}$. This is shown in Fig. 5.15 which plots normalized Fe/O vs. C/He, for intervals during the gradual SEP events, in both panels, with $T$ as symbols in the lower panel and power of $A / Q$ as symbols in the upper. 
Fig. 5.14 Source-plasma temperature is shown as a function of associated CME speed for gradual SEP events with GLE events identified (data from Reames 2016a)

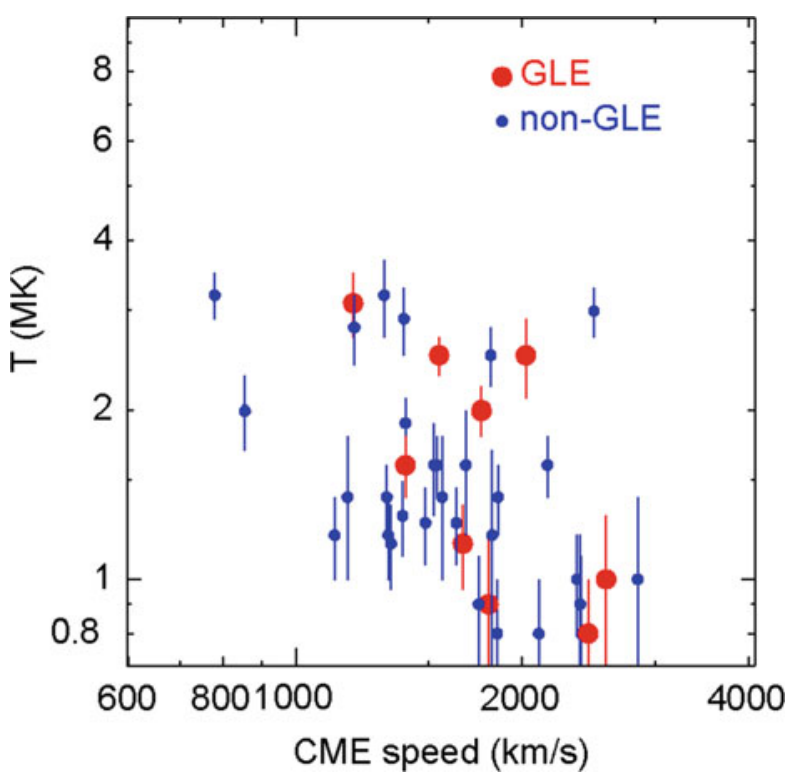

The region of abundances showing active-region temperatures $T \geq 2 \mathrm{MK}$ is immediately distinguishable, clustering in the upper left of the lower panel of Fig. 5.15. These events, which get their enhanced Fe/O from their impulsive seed population which also has enhanced He (see Sects. 5.8 and 5.9), are distinguished as open circles in the upper panel as well. Points during large events accelerated from specific temperatures of ambient coronal plasma stretch from upper right, with steep positive $A / Q$ enhancements early in the events, toward the lower center, where the $A /$ $Q$ slopes are reduced or negative, late in the same events.

The events beginning in the large gray circle in the upper right corners of the panels in Fig. 5.15 are all high-fluence, $T<2 \mathrm{MK}$ events with $>30 \mathrm{MeV}$ proton fluences above $10^{7}$ protons $\mathrm{cm}^{-2} \mathrm{sr}^{-1}$. The intense streaming protons early in these events are likely to generate waves that increase particle scattering and steepen the positive $A / Q$ dependence early in the events (e.g. Fig. 9.18), enhancing both $\mathrm{Fe} / \mathrm{O}$ and $\mathrm{C} / \mathrm{He}$. Smaller events with $T<2 \mathrm{MK}$ lack large enhancements of Fe/O. The events with $T>2 \mathrm{MK}$ are also smaller but they get their Fe/O enhancement from their impulsive seed population. Thus, gradual SEP events can become Fe-rich either by reaccelerating Fe-rich impulsive suprathermal ions or by having protons intense enough that self-generated waves preferentially transmit $\mathrm{Fe}$ and retard $\mathrm{O}$.

One might well ask: why do we use theoretical values of $Q$ vs. $T$ when there are actually direct measurements of $Q$ in some events (see Sect. 2.6)? Direct measurements can determine distributions of $Q$ which may not be isothermal and may differ somewhat from element to element. Primarily, direct measurements are rare, especially above $1 \mathrm{MeV}$, where deflection in the Earth's field is usually required (e.g. Leske et al. 1995). Also, in impulsive events, for example, $Q_{\mathrm{Fe}}$ measured at $1 \mathrm{AU}$, is observed to increase with energy at low energies (e.g. DiFabio et al. 2008), 


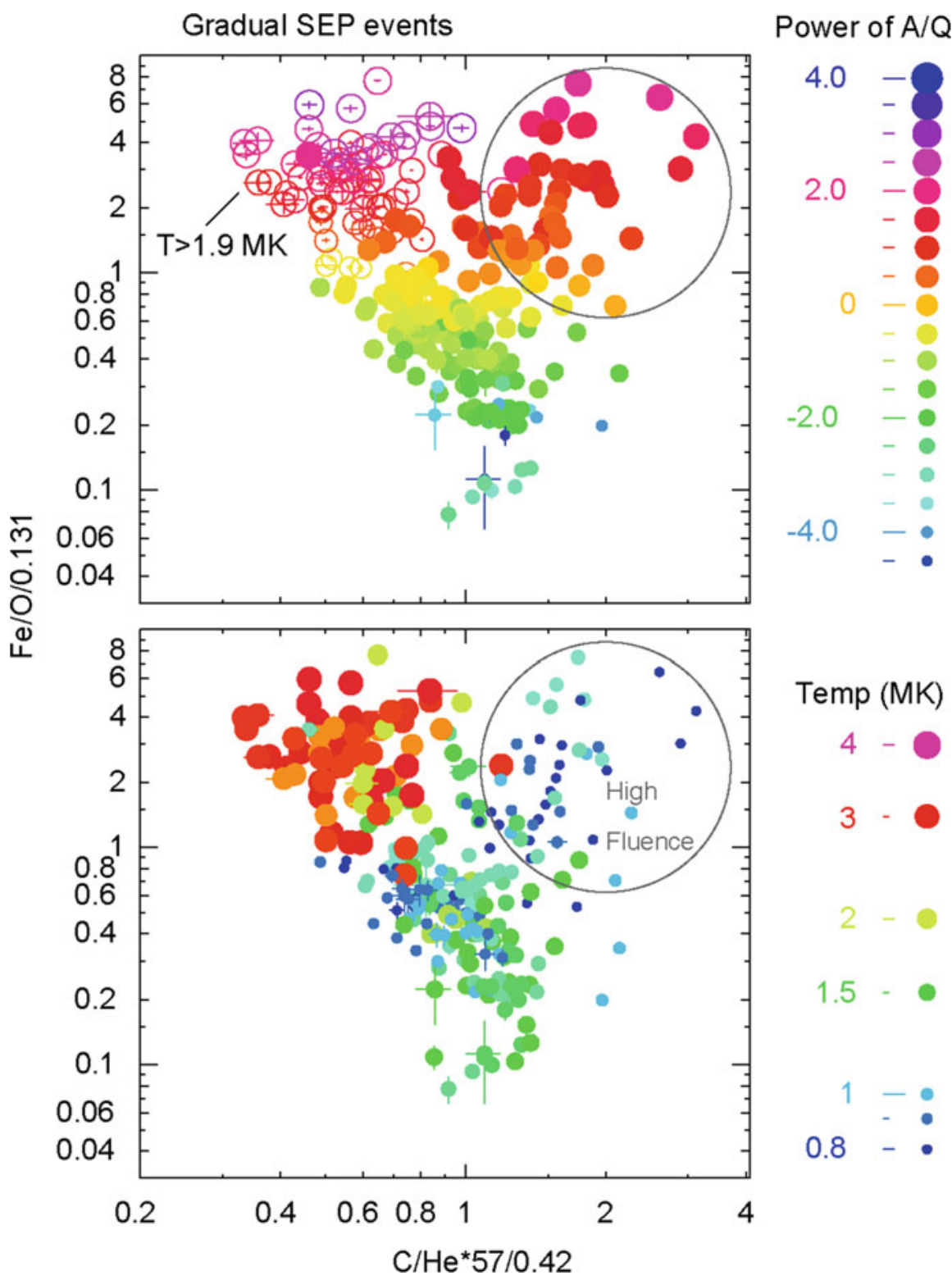

Fig. 5.15 Normalized abundance ratios $\mathrm{Fe} / \mathrm{O}$ vs. $\mathrm{He} / \mathrm{C}$ is plotted in both panels with symbol size and color representing $T$ (lower panel) and power of $A / Q$ (upper panel). (Reames 2016b (C) Springer). The large circle surrounds "big events" with high fluences of $>30 \mathrm{MeV}$ protons 
suggesting that the ions have traversed enough material after acceleration to strip them to equilibrium charges that depend only upon their velocity, i.e. they are stripped to high $Q$. The theoretical charges, obtained from abundance measurements, are more likely to be appropriate earlier, i.e. at the time of acceleration. In addition, the theoretical charges from atomic physics are available for essentially all elements we measure and abundance measurements are often available at places and times where direct measurements are not. Thus we can measure the average source plasma temperatures wherever abundance measurements are available, but, unfortunately, these measurements do not determine charge distributions, distinguish multiple sources, or assess small temperature variations among elements, as direct measurements can.

An alternative method suggested for measuring ionization states in large SEP events involves fitting the $Q / A$-dependent shapes of the intensity-time profiles using diffusion theory (Sollitt et al. 2008). The comparison of this diffusive method with the power-law techniques is presently somewhat limited, and, unfortunately, we will see in the next section that the time decay profile of SEP events is actually controlled much less by diffusion than by the expansion of a magnetic trapping volume called the reservoir.

\subsection{Spatial Distributions and the Reservoir}

As spacecraft began to probe more-distant areas of the heliosphere, it became possible to view spatial distributions of SEPs, and their time variations, within a single SEP event. While spatial gradients were expected, it was rather surprising when equal intensities of $\sim 20 \mathrm{MeV}$ protons were found over long distances of solar longitude of $\sim 180^{\circ}$ on the Pioneer spacecraft by McKibben (1972). Twenty years later equal intensities were found in radius late in large events between Ulysses at 2.5 AU and IMP 8 near Earth by Roelof et al. (1992) who named the regions "reservoirs." Reservoirs extend to Ulysses at heliolatitudes up to $>70^{\circ}, \mathrm{N}$ and $\mathrm{S}$ (Lario 2010), and they are also seen in other electron observations (Daibog et al. 2003).

The two spacecraft of the Helios mission provided another opportunity to measure the evolution of SEP events at different longitudes confirming that the longitude distribution of Fig. 2.2 was appropriate for each individual event. Figure 5.16 shows that, at widely separated spacecraft, the intensities merge with that at Helios 1 as each of the other spacecraft, Helios 2 and IMP 8, joins it in the reservoir. Spectra are identical throughout the reservoir but intensities decrease adiabatically with time as the volume of this "magnetic bottle" expands. (The drawing in the lower panel of Fig. 5.16 shows the spacecraft penetrating the CME; in reality, of course, the spacecraft are nearly stationary as the CME expands past them, but that version would be much more difficult to draw.)

If there were significant leakage from the reservoir, one would expect the highestenergy protons to leak first, since they are faster, scatter less, and encounter the 

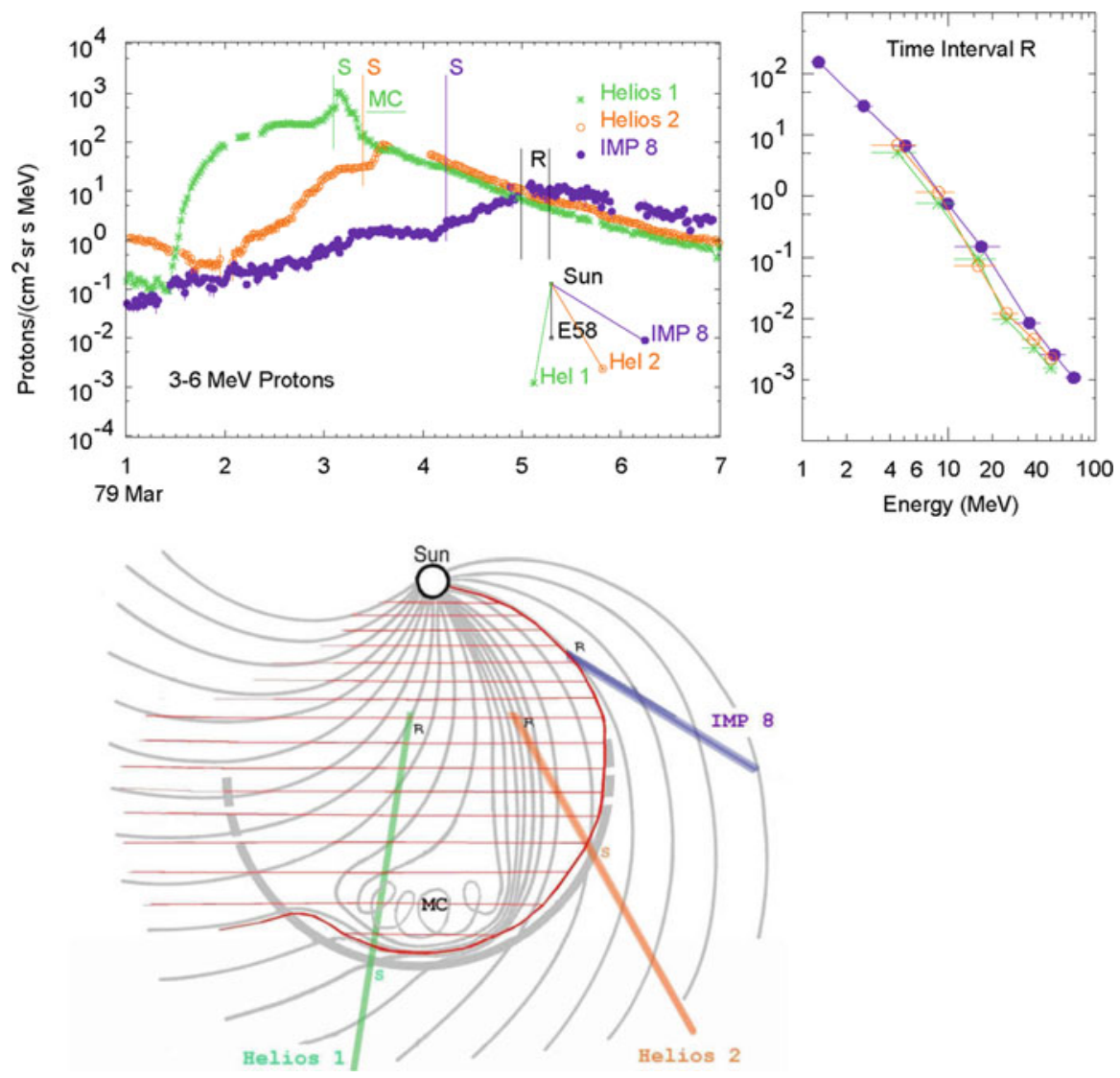

Fig. 5.16 The upper left panel shows the intensities of $3-6 \mathrm{MeV}$ protons at three spacecraft vs. time. The paths of the spacecraft into the expanding CME are shown below as they penetrate into the reservoir region (red hashing) behind the shock and CME where all intensities and spectra (upper right) are equal spatially, though they decrease with time as the trapping volume expands (Reames 2013; after Reames et al. 1997b (C) AAS)

boundary most often, but this would steepen the spectrum with time and is not observed. Thus the leakage must be minimal.

One common, but rather poor, way of comparing spatial variations is to plot peak intensity at each of three spacecraft vs. longitude and fit the three points with a parabola. Does this measure particle spread in longitude? Suppose we made such a plot with the data shown in Fig. 5.16. The intensity at Helios 1 peaks at the time of local shock passage. The intensity at Helios 2 peaks when it enters the reservoir, where it has the same intensity as Helios 1 . The intensity at IMP 8 peaks when it enters the reservoir later, where all three intensities are equal. What does the parabola defined by these three peak intensities measure? Is it the spread of the particles or the spread in the trapping volume behind the CME with time? The peaks all occur at different times and that essential timing information is lost when plotting 

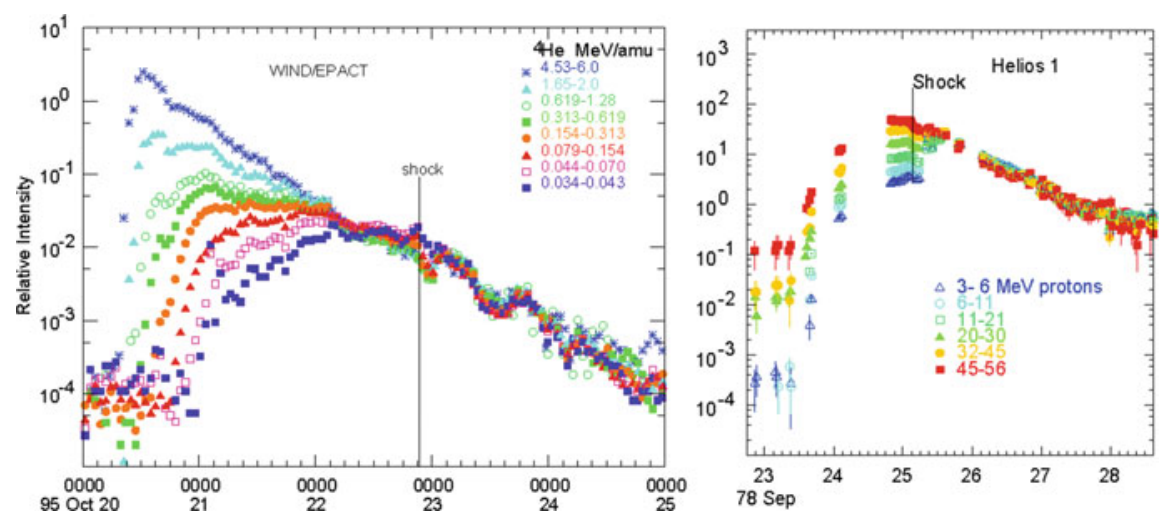

Fig. 5.17 In invariant spectral regions, particle intensities at different energies maintain the same relative normalization as a function of time, as shown for different species in two different events (Reames 2013; after Reames et al. 1997a, b C AAS)

only peak intensities vs. longitude. Isn't it more important to note that all intensities are equal when the intensity at IMP 8 peaks? It seems more productive to try to distinguish spatial and temporal effects rather than combining them.

For a single spacecraft, one way to show that spectra do not change their shape in time is to normalize the intensity-vs.-time plots at one point in time. If they stay normalized subsequently, then the spectral shapes are invariant. This is shown for two gradual SEP events in Fig. 5.17. This technique demonstrates invariance even when the spectra do not have power-law form. Multiple spacecraft at different locations can be included or abundance variations can be compared similarly.

Note that a reservoir can sometimes extend upstream of the CME and shock on the East flank, as seen in the left panel of Fig. 5.17; here the particles may be partly contained by self-amplified waves from earlier streaming or by other preexisting magnetic boundaries.

The realization that the slow decline in a gradual SEP event results from expansion of a reservoir is most important because it displaces the previous idea that slow particle spatial diffusion detained the ions to explain the decay phase of events. Actually, reservoirs are scatter free, as shown by the striking example of the little scatter-free ${ }^{3} \mathrm{He}$-rich event from Mason et al. (1989) shown as Fig. 2.3 in Sect. 2.3.4. A whole literature of fitting SEP events to diffusion theory had emerged, leading to the "Palmer (1982) consensus" that " $\lambda_{\text {回 }}=0.08-0.3$ AU over a wide range of rigidity." This is yet another example of the misapplication of diffusion theory; the intensity decline comes from the expansion of a magnetic bottle in time, not inefficient transport through space. There are no significant spatial gradients within reservoirs.

In some events we may use abundances to map variations of source plasma temperatures across the face of the shock and back into the reservoir. Figure 5.18 shows a temperature analysis that compares conditions at the STEREO B and the Wind spacecraft during an SEP event on 31 August 2012. 


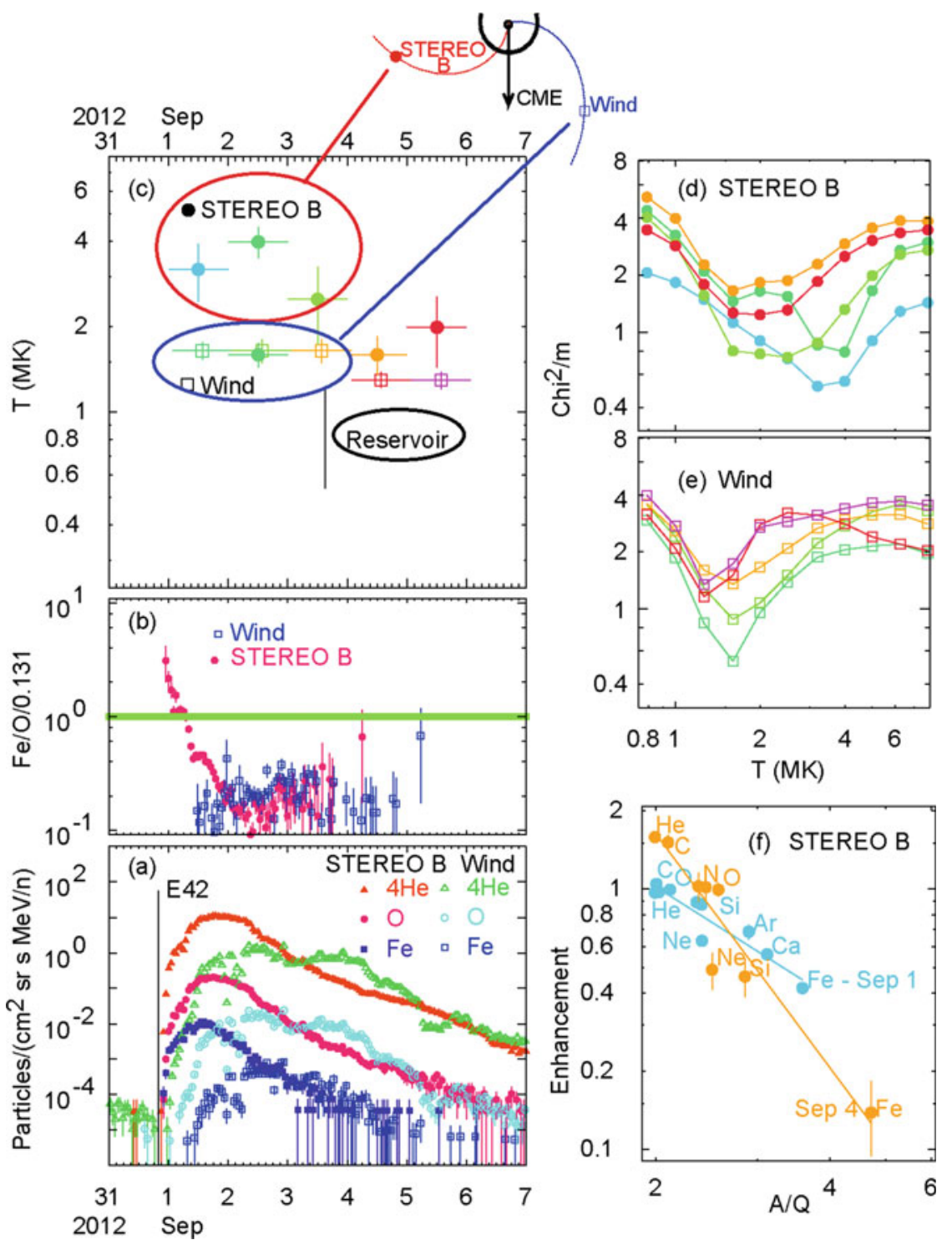

Fig. 5.18 Time variations of (a) intensities, (b) Fe/O, and (c) derived source plasma temperatures are compared at STEREO B (closed symbols) and Wind (open symbols) for the SEP event of 31 August 2012 where spacecraft locations are shown above. Probable entry of the spacecraft into a reservoir occurs sometime on 3 September as noted in (c). Curves of $\chi^{2} / m$ vs. $T$ used to select the best-fit temperatures are shown in (d) and (e) and fits to enhancements vs. $A / Q$ are shown at STEREO (f) before (September 1) and after (September 3) reservoir entry (Reames 2017a (C) Springer) 
The event in Fig. 5.18 shows the presence of hot ( 3-4 MK) source plasma at the well-connected spacecraft (STEREO B), but not an enhancement from impulsive seed particles, and ambient coronal plasma $(\sim 1.6 \mathrm{MK})$ on the flanks of the CME (at Wind). This condition persists until both spacecraft seem to have entered a reservoir, where similar intensities (Fig. 5.18a) and lower-temperature plasma (Fig. 5.18c) prevails.

Similar temperature variations across the face of the shock are possible, but less dramatic, in other events that have been measured (Reames 2017a). In one event, 23 January 2012, a spectral break disrupts the abundances, but we successfully revisit this event in Sect. 9.7. Spectral breaks are likely to make it difficult to derive temperatures from abundances; breaks are more likely above $\sim 10 \mathrm{MeV} \mathrm{amu}^{-1}$. At low energy $\left(<1 \mathrm{MeV} \mathrm{amu}^{-1}\right)$, shock waves accelerate ions far out into the heliosphere, sampling a widely varying seed population that may blur temperatures. In addition, strong suppression of low-energy spectra by the streaming limit (see Fig. 5.5) may disrupt temperature measurements at energies below $1 \mathrm{MeV} a m u^{-1}$. Thus, ion temperatures for gradual SEP events are best measured in the range of $1-10 \mathrm{MeV} \mathrm{amu}^{-1}$.

\subsubsection{Reservoirs, Loops, and Long-Duration $\gamma$ Rays}

It is important to recognize that reservoirs trap energetic ions in an expanding volume above the solar surface for a long period of time. While this population of particles tends to be mirrored by the converging magnetic fields above the corona, some undoubtedly scatter into the loss cone and plunge into the corona to produce $\gamma$ rays (just as the particles in flaring loops must do on a much faster time scale). Vestrand and Forrest (1993) observed $\gamma$-ray production spanning over $\approx 30^{\circ}$ of the Sun's surface in the large GLE of 29 September 1989. More recently, Plotnikov et al. (2017) found three events for which CMEs from behind the solar limb drove quasiperpendicular shock waves around to the footpoints of field lines on the visible disk, producing long-duration emission of $>100 \mathrm{MeV} \gamma$-rays.

Ryan (2000) discussed long-duration $\gamma$-ray events lasting an hour or more while the flare-associated X-rays died away rapidly. See, also, the long-duration $\gamma$-ray observations by Ackermann et al. (2014) and Ajello et al. (2014). Share et al. (2018) studied 29 long-duration events with $>100 \mathrm{MeV} \gamma$-rays. They concluded that the $\gamma$ rays resulted from the decay of neutral $\pi$ mesons produced by shock-accelerated $>300 \mathrm{MeV}$ SEP protons trapped in the reservoirs behind these events. Reservoirs provide an invariant spectrum of shock-accelerated ions that can bombard a large area of the solar corona with energetic SEP proton intensities that slowly decrease over many hours or days. The SEPs trapped in a reservoir may have difficulty directly penetrating the magnetic fields above active regions, and the higher-energy SEPs may begin to reach the lower solar corona only as the trapping volume widens laterally beyond the active region. In some cases studied by Share et al. (2018), the $>100 \mathrm{MeV} \gamma$-ray intensity rises slowly for several hours before it eventually begins 
to decrease, suggesting a slow increase in the area of the reservoir footprint on the Sun.

Gopalswamy et al. (2019) found a strong correlation of the duration of events with sustained $\gamma$-ray emission with the frequency and duration of associated type II radio emission. They found that the tail of the $\gamma$-ray event "seems to last until the end of the associated type II burst." Evidently both types of emission were associated with shock acceleration, as would not be too surprising if CMEs drive shocks and trap reservoirs. However, Gopalswamy et al. (2020) found an unusual geometry in the 1 September 2014 SEP event, where a shock wave was driven along an east-west flux rope, accelerating protons that plunged into the corona at the end of the flux rope, producing $\pi^{0}$ mesons and their energetic $\gamma$-ray decays. Thus, some $\gamma$-ray events are produced directly by shock-driven proton events.

Those few events where the $\gamma$-rays seem to exceed estimated production by the observed proton spectra can be explained by poor magnetic connection. For example, a case where the nose of the shock is seen to be driven toward high latitude above the ecliptic so the Earth only samples an inadequate soft proton spectrum from the flank of the shock. All of the $\gamma$-ray events can be reasonably explained by shockaccelerated protons (Gopalswamy et al. 2019, 2020).

\subsection{Non-thermal Variations: Impulsive vs. Gradual SEPs}

Knowing the source-plasma temperatures allows us to compare impulsive and gradual SEP events from the same temperature source-i.e. impulsive SEP ions and impulsive seed particles. Figure 5.19 compares the normalized abundances of $\mathrm{O} / \mathrm{C}$ vs. $\mathrm{C} / \mathrm{He}$ for impulsive and gradual SEP events plotted at the same scale. The impulsive events have been limited to those with modest $<20 \%$ statistical errors in the ratios and the gradual events at $T \geq 2 \mathrm{MK}$ come from impulsive seed ions.

Especially at a temperature of 3.2 MK (red symbols in Fig. 5.19) the elements $\mathrm{He}$ and $\mathrm{C}$ are likely to be fully ionized with $A / \mathrm{Q}=2$ and $\mathrm{O}$ is nearly so (as seen in Fig. 5.11). Thus the ratios should be unaltered source abundances for both populations. However the dashed line also shows that the normalization is wrong for $\mathrm{C} / \mathrm{He}$ since the central mean should be at 1.0 if properly normalized. This suggests that the reference abundance $\mathrm{He} / \mathrm{O}$ should be 91 rather than 57 . This would bring $\mathrm{He}$ in somewhat better alignment with other high-FIP elements on a FIP plot (Fig. 1.6) and is shown as a red open circle on that figure.

More significantly, the spread in the distribution of gradual events is much smaller than that of impulsive events in Fig. 5.19. The spread in the impulsive events must come from non-thermal abundance variations in the local plasma of jets where the magnetic reconnection is occurring. However, neither wave-particle interactions nor magnetic reconnection can alter $\mathrm{C} / \mathrm{He}$ when both elements have $\mathrm{A} /$ $Q=2.0$. If the shock wave of a gradual SEP event were accelerating only suprathermal ions from a single impulsive source, we would expect the same non-thermal distribution for gradual events that we see for impulsive events. This is not the case. 


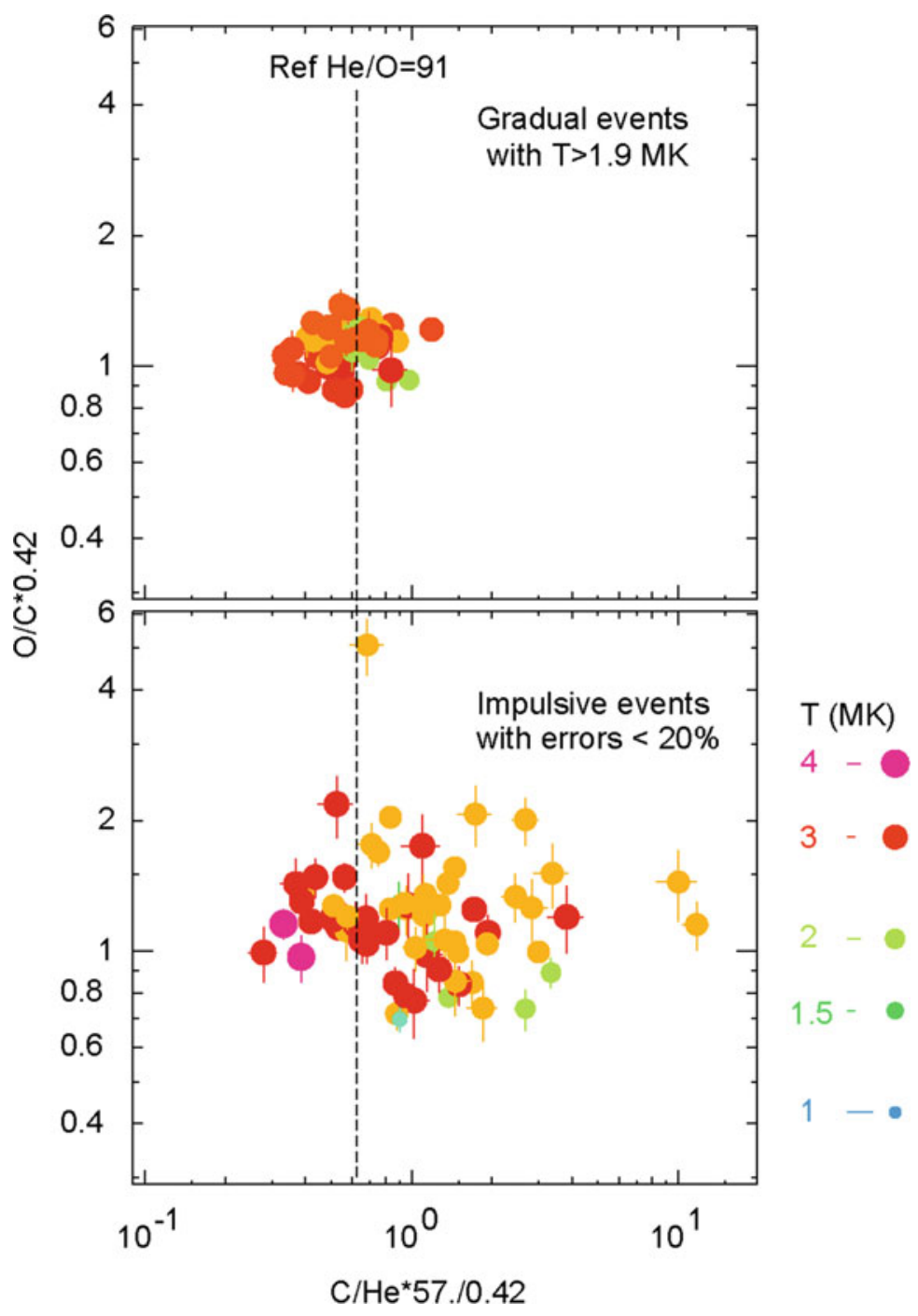

Fig. 5.19 Enhancements of $\mathrm{O} / \mathrm{C}$ vs. $\mathrm{C} / \mathrm{He}$ are compared, for gradual events with $T \geq 2.0 \mathrm{MK}$ (upper panel) and impulsive events with $<20 \%$ errors so that the spread is not from statistical errors (lower panel). Both panels are plotted at the same scale and $T$ is indicated by the size and color of the symbols. (1) The distribution is much smaller for the gradual events. (2) The median of the distribution of $\mathrm{C} / \mathrm{He}$ for the gradual events, shown as a dashed line, implies a reference value for $\mathrm{He} / \mathrm{O}$ of 91 rather than 57 (Reames 2016b (C) Springer)

As the shock in a gradual event passes over an active region, it must average contributions (1) from impulsive suprathermal ions, which have enhancements in $\mathrm{Fe} / \mathrm{O}$ and ${ }^{3} \mathrm{He} /{ }^{4} \mathrm{He}$, for example, and perhaps (2) from ions in the ambient $\sim 3 \mathrm{MK}$ plasma, which have no such enhancements. Ko et al. (2013) found that Fe-rich gradual SEP events were commonly connected to active regions. The result of the 
two contributions is to reduce the enhancements, as observed, and somewhat reduced distributions in the spread of abundance ratios, more like those in the upper panel of Fig. 5.19.

However, if we really expect to reduce the spread of the distributions as seen in the gradual events, we need to average over several small jets producing impulsive SEP events rather than only one; $n$ events will reduce the spread by a factor of $\sqrt{ } n$. Thus a pool of 10 contributing events with $30 \%$ abundance variations would reduce the average variation of the seed population below 10\%. It is likely that the number of small impulsive SEP events in an active region increases as the event size decreases, contributing a fairly steady flow of impulsive suprathermal ions; each temporarily contributes to the potential seed population before it diminishes. Based on the increasing number of flares with decreasing size, Parker (1988) proposed that a large number of small nanoflares could actually heat the corona. We need only a small increase in the number of jets producing impulsive SEP events that are too small to resolve as separate events, yet adequate to contribute to the seed population in the pool of impulsive suprathermal ions above a solar active region which may be subsequently sampled and averaged by a shock wave. Thus, no single impulsive event determines the seed population for acceleration by the shock wave in a subsequent gradual SEP event.

Many small jets (i.e. microjets or nanojets?) probably also contribute to the periods of persistent ${ }^{3} \mathrm{He}$ seen by Wiedenbeck et al. (2008), of long-lived and recurrent sources (Bučík et al. 2014, 2015; Chen et al. 2015), often generated by "geysers" that produce multiple jets (e.g. Paraschiv and Donea 2019), and, of course, to the substantial persistent ${ }^{3} \mathrm{He}$ abundances below $1 \mathrm{MeV} \mathrm{amu}^{-1}$ in the seed population directly observed at 1 AU "upstream" region (labeled period A) in Fig. 2.8 where ${ }^{3} \mathrm{He}$-rich, Fe-rich periods in the seed population are seen on 20 and 21 June 2000 and Fe-rich periods (labeled B) again on 24 and 25 June after the shock wave of interest (e.g. Desai et al. 2003).

Source-plasma temperatures provide a powerful new tool for the comparative study of SEP events.

\subsection{The Abundance of He and the FIP Effect}

The preceding section suggests that $\mathrm{He} / \mathrm{O}$, in both impulsive SEP events and in gradual events that re-accelerated impulsive $\sim 3 \mathrm{MK}$ plasma, may be similar to the average value of $\mathrm{He} / \mathrm{O}$ in the solar wind, while $\mathrm{He} / \mathrm{O}$ can be much lower in gradual events involving cooler $(<2 \mathrm{MK})$ ambient source plasma. He variations should not come as a complete surprise since variations of $\mathrm{He} / \mathrm{O}$ are also seen in the solar wind as functions of time and of solar-wind speed (Collier et al. 1996; Bochsler 2007; Rakowsky and Laming 2012). The study of the source-plasma temperatures of gradual SEP events (Reames 2016a) originally assumed the source had $\mathrm{He} / \mathrm{O}=57$ 

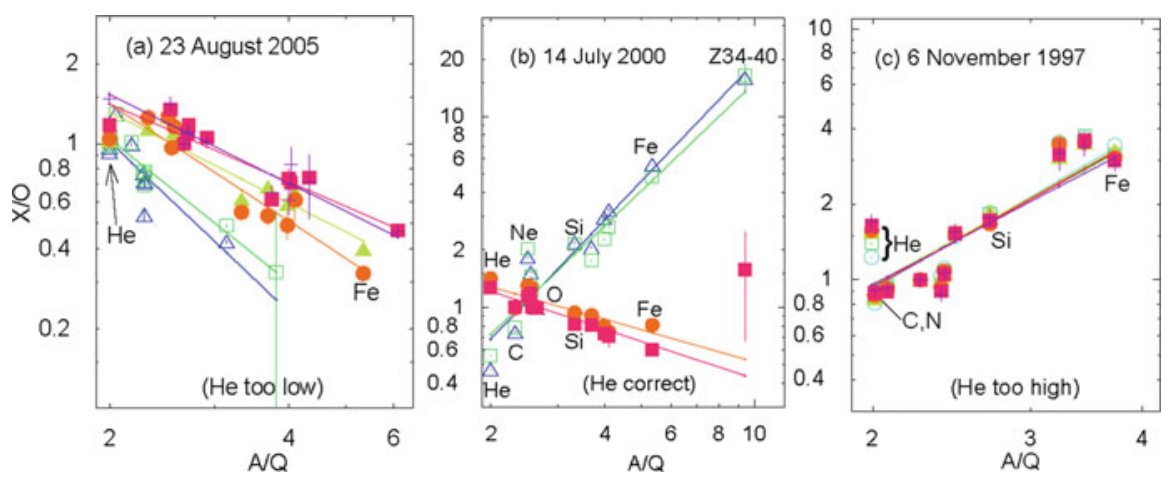

Fig. 5.20 Best fits to enhancement vs. $A / Q$ assuming the source $\mathrm{He} / \mathrm{O}=57$ at times during three gradual SEP events (Reames 2017b). For (a) the He enhancement is too low, for (c) it is too high, and for (b) it is approximately correct (i.e. in agreement with the fit of other ions) for both enhanced and suppressed periods of $\mathrm{Fe} / \mathrm{O}$

as derived from the SEP average. For some events, however, He fell far from the least-squares fits of enhancements vs. $A / Q$ for all the other elements, as seen in Fig. 5.20a, c.

For the SEP event of 23 August 2005 in (a), for which $T=1.0 \pm 0.2 \mathrm{MK}$, a lower value of source $\mathrm{He} / \mathrm{O} \approx 45$ would lift the enhancement of He to the fit lines. For the 6 November event (c), with $T=2.5 \pm 0.2 \mathrm{MK}$, a value of source $\mathrm{He} / \mathrm{O} \approx 100$ would drive He down to the fit lines. However, for the event of 14 July 2000, with $T=1.2 \pm 0.2$, the average value of source $\mathrm{He} / \mathrm{O} \approx 57$, or slightly less, seems quite reasonable for both ascending and descending enhancement periods (Reames 2017b). He, the element with the highest FIP (24.6 eV), is the slowest element to ionize during its transit of the chromosphere into the corona, so its abundance is most likely to lag that of the other elements (Laming 2009, 2015); thus, low values of $\mathrm{He} / \mathrm{O}$ may indicate newly arriving coronal ions while high values suggest established abundances.

The distribution vs. $T$ of the values of the source $\mathrm{He} / \mathrm{O}$ that would be required for the observed $\mathrm{He} / \mathrm{O}$ to fit the power-law defined by the $Z>2$ elements in each 8 -h interval is shown in Fig. 5.21. The reaccelerated impulsive-event material at $\approx 3 \mathrm{MK}$ supplies the higher reference $\mathrm{He} / \mathrm{O}$ material.

Understanding the possible slow ionization of He helps resolve differences in $\mathrm{He} / \mathrm{O}$ between SEPs and the solar wind, although the remaining differences, especially in $\mathrm{C} / \mathrm{O}$, show us that SEPs and the solar wind have different FIP patterns, hence different origins at the base of the solar corona, as we will find in Chap. 8. This will show that SEPs are not merely accelerated solar wind. They are a particle population with unique coronal origin. 


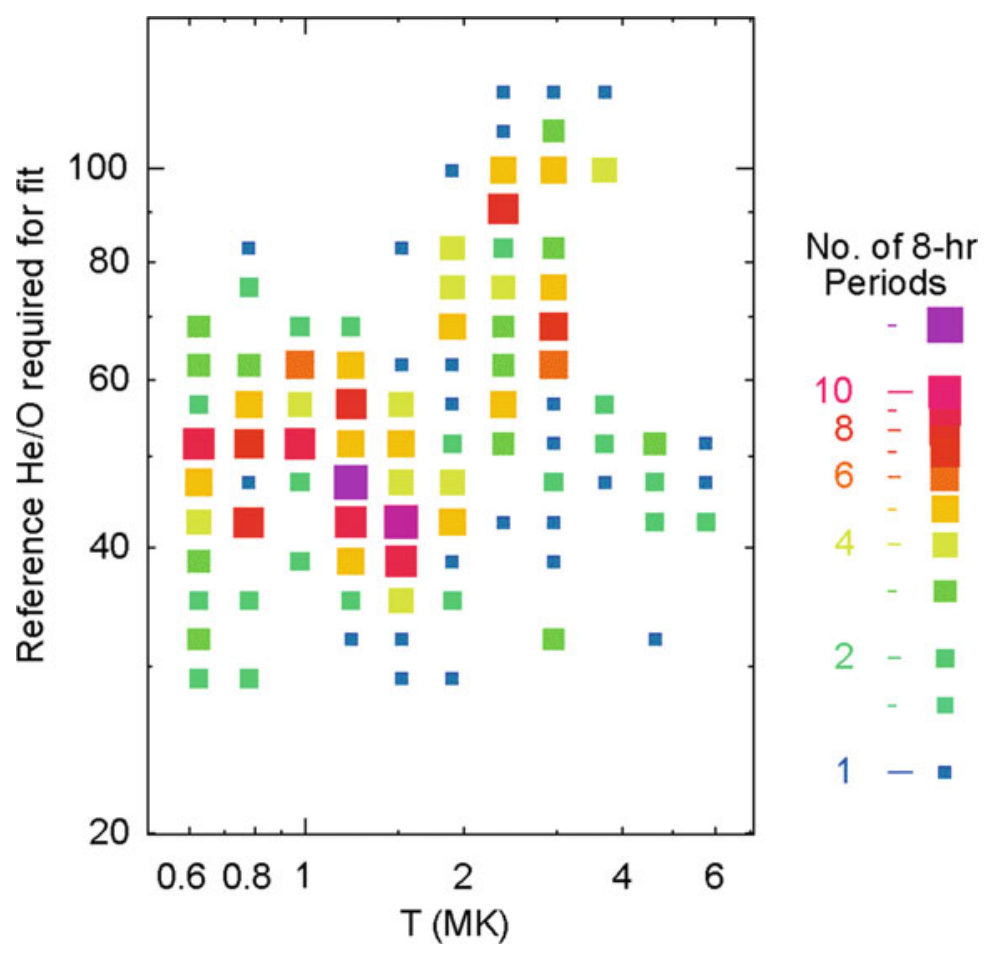

Fig. 5.21 A histogram for 8-h intervals in gradual SEP events of the source value of $\mathrm{He} / \mathrm{O}$ required for the $\mathrm{He} / \mathrm{O}$ abundance enhancement to fit the power-law distribution defined by the elements with $Z>2$, as a function of the best-fit source-plasma temperature (Reames 2017b (C) Springer)

\subsection{Open Questions}

This section suggests open questions that might be addressed in future research.

1. What can cause the large non-thermal spread of abundances such as $\mathrm{C} / \mathrm{He}$ in impulsive SEP events in Fig. 5.19 when both He and $\mathrm{C}$ should be fully ionized? Does source depth in the corona matter?

2. How well do SEP-derived temperatures correlate with differently-determined coronal temperatures near the observer's magnetic footpoint early in a gradual SEP event?

3. How can reservoirs contain particles of all energies with such apparently equal efficiency? How do they attain uniformity of intensities across longitudes when the particles upstream of the shock do not? Is diffusion along the turbulent shock front a factor? 
4. In principle, a shock could accelerate 1-MK plasma at one longitude and 3-MK plasma at another longitude. Only one clear case is seen. Is there enough lateral transport in and behind the shock to mix SEPs from these sources in the reservoir late in events?

5. Which events have reservoirs and which do not? What is the difference?

6. What happens when the energy in SEPs exceeds the energy in $B$ at a shock, especially a quasi-perpendicular shock? Can it do so? Does acceleration cease?

7. Measurements on a spacecraft nearer the Sun can improve SEP onset timing by removing the blurring effect of scattering during transport. How does the SEP onset SPR time at 10 -second resolution compare with X-ray and $\gamma$-ray-line onsets, type II burst timing, and shock observations? Note that intensities may vary as $\sim r^{-3}$, causing extremely high rates in instruments. To what extent does electron and ion source timing differ in gradual SEP events? In impulsive events?

8. Discrete ionization states affect the assignment of source-plasma temperatures. ${ }^{12} \mathrm{C}^{+5}$ is enhanced but ${ }^{12} \mathrm{C}^{+6}$ is not; treating $Q$ as 5.5 is approximate. $A /<Q>$ is not the same as $\left\langle A / Q>\right.$. Then there is ${ }^{13} \mathrm{C}$ which always has high $A / Q$. Can we improve the estimates of $T$ ?

\section{References}

Ackermann, M., et al.: High-energy gamma-ray emission from solar flares: summary of Fermi large area telescope detections and analysis of two M-class flares. Astrophys. J. 787, 15 (2014). https://doi.org/10.1088/0004-637X/787/1/15

Afanasiev, A., Battarbee, M., Vainio, R.: Self-consistent Monte Carlo simulations of proton acceleration in coronal shocks: Effect of anisotropic pitch-angle scattering of particles. Astron. Astrophys. 584, 81 (2016). https://doi.org/10.1051/0004-6361/201526750

Afanasiev, A., Vainio, R., Rouillard, A.P., Battarbee, M., Aran, A., Zucca, P.: Modelling of proton acceleration in application to a ground level enhancement. Astron. Astrophys. 614, A4 (2018). https://doi.org/10.1051/0004-6361/201731343

Ajello, M., et al.: Impulsive and long duration high-energy gamma-ray emission from the very bright 2012 March 7 solar flares. Astrophys. J. 789, 20 (2014). https://doi.org/10.1088/0004$637 \mathrm{X} / 789 / 1 / 20$

Axford, W.I.: Acceleration of cosmic rays by shock waves. Proc. 17th Int. Cos. Ray Conf. (Paris). 12, 155 (1981)

Bell, A.R.: The acceleration of cosmic rays in shock fronts. I, Monthly Notices Roy. Astron. Soc. 182, 147 (1978a). https://doi.org/10.1093/mnras/182.2.147

Bell, A.R.: The acceleration of cosmic rays in shock fronts. II, Monthly Notices Roy. Astron. Soc. 182, 443 (1978b). https://doi.org/10.1093/mnras/182.3.443

Berdichevsky, D.B., Szabo, A., Lepping, R.P., Viñas, A.F., Mariana, F.: Interplanetary fast shocks and associated drivers observed through the 23rd solar minimum by Wind over its first 2.5 years. J. Geophys. Res. 105, 27289 (2000). https://doi.org/10.1029/1999JA000367

Bochsler, P.: Solar abundances of oxygen and neon derived from solar wind observations. Astron. Astrophys. 471, 315 (2007). https://doi.org/10.1051/0004-6361:20077772

Breneman, H.H., Stone, E.C.: Solar coronal and photospheric abundances from solar energetic particle measurements. Astrophys. J. Lett. 299, L57 (1985). https://doi.org/10.1086/184580

Bučík, R., Innes, D.E., Mall, U., Korth, A., Mason, G.M., Gómez-Herrero, R.: Multi-spacecraft observations of recurrent ${ }^{3} \mathrm{He}$-rich solar energetic particles. Astrophys. J. 786, 71 (2014). https:// doi.org/10.1088/0004-637X/786/1/71 
Bučík, R., Innes, D.E., Chen, N.H., Mason, G.M., Gómez-Herrero, R., Wiedenbeck, M.E.: Longlived energetic particle source regions on the Sun. J. Phys. Conf. Ser. 642, 012002 (2015). https://doi.org/10.1088/1742-6596/642/1/012002

Chen, N.H., Bučík, R., Innes, D.E., Mason, G.M.: Case studies of multi-day ${ }^{3}$ He-rich solar energetic particle periods. Astron. Astrophys. 580, 16 (2015). https://doi.org/10.1051/0004$6361 / 201525618$

Collier, M.R., Hamilton, D.C., Gloeckler, G., Bochsler, P., Sheldon, R.B.: Neon-20, oxygen-16, and helium-4 densities, temperatures, and suprathermal tails in the solar wind determined with WIND/MASS. Geophys. Res. Lett. 23, 1191 (1996). https://doi.org/10.1029/96GL00621

Daibog, E.I., Stolpovskii, V.G., Kahler, S.W.: Invariance of charged particle time profiles at late stages of SCR events from the data of multisatellite observations. Cosm. Res. 41, 128 (2003)

Desai, M.I., Giacalone, J.: Large gradual solar energetic particle events. Living Rev. Solar Phys. 13, 3 (2016). https://doi.org/10.1007/s41116-016-0002-5

Desai, M.I., Mason, G.M., Dwyer, J.R., Mazur, J.E., Gold, R.E., Krimigis, S.M., Smith, C.W., Skoug, R.M.: Evidence for a suprathermal seed population of heavy ions accelerated by interplanetary shocks near 1 AU. Astrophys. J. 588, 1149 (2003). https://doi.org/10.1086/ 374310

Desai, M.I., Mason, G.M., Wiedenbeck, M.E., Cohen, C.M.S., Mazur, J.E., Dwyer, J.R., Gold, R. E., Krimigis, S.M., Hu, Q., Smith, C.W., Skoug, R.M.: Spectral properties of heavy ions associated with the passage of interplanetary shocks at 1 AU. Astrophys. J. 661, 1156 (2004). https://doi.org/10.1086/422211

DiFabio, R., Guo, Z., Möbius, E., Klecker, B., Kucharek, H., Mason, G.M., Popecki, M.: Energydependent charge states and their connection with ion abundances in impulsive solar energetic particle events. Astrophys. J. 687, 623 (2008). https://doi.org/10.1086/591833

Ellison, D., Ramaty, R.: Shock acceleration of electrons and ions in solar flares. Astrophys. J. 298, 400 (1985). https://doi.org/10.1086/163623

Gopalswamy, N., Makela, P., Yashiro, S., Lara, A., Akiyama, S., Xie, H.: On the shock source of sustained gamma-ray emission from the sun. J. Phys. Conf. Ser. 1332, 1 (2019). https://doi.org/ 10.1088/1742-6596/1332/1/012004

Gopalswamy, N., Mäkelä, P., Yashiro, S., Akiyama, S., Xie, H., Thakur, N.: Source of energetic protons in the 2014 September 1 sustained gamma-ray emission event. Sol. Phys. 295, 18 (2020). https://doi.org/10.1007/s11207-020-1590-8

Hu, J., Li, G., Ao, X., Verkhoglyadova, O., Zank, G.: Modeling particle acceleration and transport at a 2D CME-driven shock. J. Geophys. Res. 122, 10,938 (2017). https://doi.org/10.1002/ 2017JA024077

Hu, J., Li, G., Fu, S., Zank, G., Ao, X.: Modeling a single SEP event from multiple vantage points using the iPATH model. Astrophys. J. Lett. 854, L19 (2018). https://doi.org/10.3847/2041$8213 /$ aaabc 1

Jones, F.C., Ellison, D.E.: The plasma physics of shock acceleration. Space Sci. Rev. 58, 259 (1991). https://doi.org/10.1007/BF01206003

Ko, Y.-K., Tylka, A.J., Ng, C.K., Wang, Y.-M., Dietrich, W.F.: Source regions of the interplanetary magnetic field and variability in heavy-ion elemental composition in gradual solar energetic particle events. Astrophys. J. 776, 92 (2013). https://doi.org/10.1088/0004-637X/776/2/92

Laming, J.M.: Non-WKB models of the first ionization potential effect: implications for solar coronal heating and the coronal helium and neon abundances. Astrophys. J. 695, 954 (2009). https://doi.org/10.1088/0004-637X/695/2/954

Laming, J.M.: The FIP and inverse FIP effects in solar and stellar coronae. Living Rev. Sol. Phys. 12, 2 (2015). https://doi.org/10.1007/lrsp-2015-2

Lario, D.: Heliospheric energetic particle reservoirs: Heliospheric energetic particle reservoirs: Ulysses and ACE 175-315 keV electron observations. Proc. 12th Solar Wind Conf. AIP Conf. Proc. 1216, 625 (2010). https://doi.org/10.1063/1.3395944

Lario, D., Decker, R.B.: The energetic storm particle event of October 20, 1989. Geophys. Res. Lett. 29, 1393 (2002). https://doi.org/10.1029/2001GL014017 
Lee, M.A.: Coupled hydromagnetic wave excitation and ion acceleration at interplanetary traveling shocks. J. Geophys. Res. 88, 6109 (1983). https://doi.org/10.1029/JA088iA08p06109

Lee, M.A.: Coupled hydromagnetic wave excitation and ion acceleration at an evolving coronal/ interplanetary shock. Astrophys. J. Suppl. 158, 38 (2005). https://doi.org/10.1086/428753

Lee, M.A., Mewaldt, R.A., Giacalone, J.: Shock acceleration of ions in the heliosphere. Space Sci. Rev. 173, 247 (2012). https://doi.org/10.1007/s11214-012-9932-y

Leske, R.A., Cummings, J.R., Mewaldt, R.A., Stone, E.C., von Rosenvinge, T.T.: Measurements of the ionic charge states of solar energetic particles using the geomagnetic field. Astrophys. J. 452, L149 (1995). https://doi.org/10.1086/309718

Li, G., Zank, G.P., Rice, W.K.M.: Acceleration and transport of heavy ions at coronal mass ejection-driven shocks. J. Geophys. Res. 110, 6104 (2005). https://doi.org/10.1029/ 2004JA010600

Li, G., Shalchi, A., Ao, X., Zank, G., Verkhoglyadova, O.P.: Particle acceleration and transport at an oblique CME-driven shock. Adv. Space Res. 49, 1067 (2012). https://doi.org/10.1016/j.asr. 2011.12.027

Mason, G.M., Ng, C.K., Klecker, B., Green, G.: Impulsive acceleration and scatter-free transport of about $1 \mathrm{MeV}$ per nucleon ions in ${ }^{3} \mathrm{He}$-rich solar particle events. Astrophys. J. 339, 529 (1989). https://doi.org/10.1086/167315

Mazzotta, P., Mazzitelli, G., Colafrancesco, S., Vittorio, N.: Ionization balance for optically thin plasmas: rate coefficients for all atoms and ions of the elements $\mathrm{H}$ to Ni. Astron. Astrophys Suppl. 133, 403 (1998). https://doi.org/10.1051/aas:1998330

McKibben, R.B.: Azimuthal propagation of low-energy solar-flare protons as observed from spacecraft very widely separated in solar azimuth. J. Geophys. Res. 77, 3957 (1972). https:// doi.org/10.1029/JA077i022p03957

Melrose, D.B.: Plasma astrophysics. Gordon and Breach, New York (1980)

Melrose, D.B., Pope, M.H.: Diffusive shock acceleration by multiple shocks. Proc. Astron. Soc. Au. 10, 222 (1993)

Mewaldt, R.A., Looper, M.D., Cohen, C.M.S., Haggerty, D.K., Labrador, A.W., Leske, R.A., Mason, G.M., Mazur, J.E., von Rosenvinge, T.T.: Energy spectra, composition, other properties of ground-level events during solar cycle 23. Space Sci. Rev. 171, 97 (2012). https://doi.org/10. 1007/s11214-012-9884-2

$\mathrm{Ng}$, C.K., Reames, D.V.: Focused interplanetary transport of approximately $1 \mathrm{MeV}$ solar energetic protons through self-generated Alfven waves. Astrophys. J. 424, 1032 (1994). https://doi.org/ $10.1086 / 173954$

Ng, C.K., Reames, D.V.: Pitch angle diffusion coefficient in an extended quasi-linear theory. Astrophys. J. 453, 890 (1995). https://doi.org/10.1086/176449

$\mathrm{Ng}$, C.K., Reames, D.V.: Shock acceleration of solar energetic protons: the first 10 minutes. Astrophys. J. Lett. 686, L123 (2008). https://doi.org/10.1086/592996

$\mathrm{Ng}$, C.K., Reames, D.V., Tylka, A.J.: Effect of proton-amplified waves on the evolution of solar energetic particle composition in gradual events. Geophys. Res. Lett. 26, 2145 (1999). https:// doi.org/10.1029/1999GL900459

Ng, C.K., Reames, D.V., Tylka, A.J.: Modeling shock-accelerated solar energetic particles coupled to interplanetary Alfvén waves. Astrophys. J. 591, 461 (2003). https://doi.org/10.1086/375293

Ng, C.K., Reames, D.V., Tylka, A.J.: Solar energetic particles: shock acceleration and transport through self-amplified waves. AIP Conf. Proc. 1436, 212 (2012). https://doi.org/10.1063/1. 4723610

Palmer, I.D.: Transport coefficients of low-energy cosmic rays in interplanetary space. Rev. Geophys. Space Phys. 20, 335 (1982)

Paraschiv, A.R., Donea, A.: On solar recurrent coronal jets: coronal geysers as sources of electron beams and interplanetary type-III radio bursts. Astrophys. J. 873, 110 (2019). https://doi.org/10. 3847/1538-4357/ab04a6

Parker, E.N.: Interplanetary dynamical processes. Interscience, New York (1963) 
Parker, E.N.: Nanoflares and the solar X-ray corona. Astrophys. J. 330, 474 (1988). https://doi.org/ $10.1086 / 166485$

Plotnikov, I., Rouillard, A., Share, G.: The magnetic connectivity of coronal shocks to the visible disk during long-duration gamma-ray flares. Astron. Astrophys. 608, 43 (2017). https://doi.org/ 10.1051/0004-6361/201730804. (arXiv:1703.07563)

Post, D.E., Jensen, R.V., Tarter, C.B., Grasberger, W.H., Lokke, W.A.: Steady-state radiative cooling rates for low-density, high temperature plasmas. At. Data Nucl. Data Tables. 20, 397 (1977). https://doi.org/10.1016/0092-640X(77)90026-2

Rakowsky, C.E., Laming, J.M.: On the Origin of the slow speed solar wind: helium abundance variations. Astrophys. J. 754, 65 (2012). https://doi.org/10.1088/0004-637X/754/1/65

Raouafi, N.E., Patsourakos, S., Pariat, E., Young, P.R., Sterling, A.C., Savcheva, A., Shimojo, M., Moreno-Insertis, F., DeVore, C.R., Archontis, V., et al.: Solar coronal jets: observations, theory, and modeling. Space Sci. Rev. 201, 1 (2016). https://doi.org/10.1007/s11214-016-0260-5. (arXiv:1607.02108)

Reames, D.V.: Acceleration of energetic particles by shock waves from large solar flares. Astrophys. J. Lett. 358, L63 (1990). https://doi.org/10.1086/185780

Reames, D.V.: Particle energy spectra at traveling interplanetary shock waves. Astrophys. J. 757, 93 (2012). https://doi.org/10.1088/0004-637X/757/1/93

Reames, D.V.: The two sources of solar energetic particles. Space Sci. Rev. 175, 53 (2013). https:// doi.org/10.1007/s11214-013-9958-9

Reames, D.V.: Element abundances in solar energetic particles and the solar corona. Sol. Phys. 289, 977 (2014). https://doi.org/10.1007/s11207-013-0350-4

Reames, D.V.: Temperature of the source plasma in gradual solar energetic particle events. Sol. Phys. 291, 911 (2016a). https://doi.org/10.1007/s11207-016-0854-9. (arXiv: 1509.08948)

Reames, D.V.: The origin of element abundance variations in solar energetic particles. Sol. Phys. 291, 2099 (2016b). https://doi.org/10.1007/s11207-016-0942-x. (arXiv: 1603.06233)

Reames, D.V.: Spatial distribution of element abundances and ionization states in solar energeticparticle events. Sol. Phys. 292, 133 (2017a). https://doi.org/10.1007/s11207-017-1138-8. (arXiv 1705.07471)

Reames, D.V.: The abundance of helium in the source plasma of solar energetic particles. Sol. Phys. 292, 156 (2017b). https://doi.org/10.1007/s11207-017-1173-5. (arXiv: 1708.05034)

Reames, D.V., Ng, C.K.: Streaming-limited intensities of solar energetic particles on the intensity plateau. Astrophys. J. 722, 1286 (2010). https://doi.org/10.1088/0004-637X/723/2/1286

Reames, D.V., Barbier, L.M., von Rosenvinge, T.T., Mason, G.M., Mazur, J.E., Dwyer, J.R.: Energy spectra of ions accelerated in impulsive and gradual solar events. Astrophys. J. 483, 515 (1997a). https://doi.org/10.1086/310845

Reames, D.V., Kahler, S.W., Ng, C.K.: Spatial and temporal invariance in the spectra of energetic particles in gradual solar events. Astrophys. J. 491, 414 (1997b). https://doi.org/10.1086/ 304939

Reames, D.V., Ng, C.K., Tylka, A.J.: Initial time dependence of abundances in solar particle events. Astrophys. J. Lett. 531, L83 (2000). https://doi.org/10.1086/319810

Reames, D.V., Ng, C.K., Berdichevsky, D.: Angular distributions of solar energetic particles. Atrophys. J. 550, 1064 (2001). https://doi.org/10.1086/319810

Rice, W.K.M., Zank, G.P., Li, G.: Particle acceleration and coronal mass ejection driven shocks: shocks of arbitrary strength. J. Geophys. Res. 108, 1369 (2003). https://doi.org/10.1029/ 2002JA009756

Roelof, E.C.: Propagation of solar cosmic rays in the interplanetary magnetic field. In: Ögelman, H., Wayland, J.R. (eds.) Lectures in high-energy astrophysics. NASA SP-199, Washington, DC (1969)

Roelof, E.C., Gold, R.E., Simnett, G.M., Tappin, S.J., Armstrong, T.P., Lanzerotti, L.J.: Low-energy solar electrons and ions observed at ULYSSES February-April, 1991 - The inner heliosphere as a particle reservoir. Geophys. Res. Lett. 19, 1247 (1992). https://doi.org/10.1029/ 92GL01312 
Rouillard, A., Sheeley Jr., N.R., Tylka, A., Vourlidas, A., Ng, C.K., Rakowski, C., Cohen, C.M.S., Mewaldt, R.A., Mason, G.M., Reames, D.V., et al.: The longitudinal properties of a solar energetic particle event investigated using modern solar imaging. Astrophys. J. 752, 44 (2012). https://doi.org/10.1088/0004-637X/752/1/44

Ryan, J.M.: Long-duration solar gamma-ray flares. Space Sci. Rev. 93, 581 (2000)

Sandroos, A., Vainio, R.: Simulation results for heavy ion spectral variability in large gradual solar energetic particle events. Astrophys. J. 662, L127 (2007). https://doi.org/10.1086/519378

Share, G.H., Murphy, R.J., White, S.M., Tolbert, A.K., Dennis, B.R., Schwarz, R.A., Smart, D.F., Shea, M.A.: Characteristics of late-phase $>100 \mathrm{MeV} \gamma$-ray emission in solar eruptive events. Astrophys. J. 869, 182 (2018). https://doi.org/10.3847/1538-4357/aaebf7

Sollitt, L.S., Stone, E.C., Mewaldt, R.A., Cohen, C.M.S., Cummings, A.C., Leske, R.A., Wiedenbeck, M.E., von Rosenvinge, T.T.: A novel technique to infer ionic charge states of solar energetic particles. Astrophys. J. 679, 910 (2008). https://doi.org/10.1086/587121

Stix, T.H.: The theory of plasma waves. McGraw-Hill, New York (1962)

Stix, T.H.: Waves in plasmas. AIP, New York (1992)

Strauss, R.D., le Roux, J.A.: Solar energetic particle propagation in wave turbulence and the possibility of wave generation. Astrophys. J. 872, 125 (2019). https://doi.org/10.3847/15384357/aafe02

Tan, L.C., Reames, D.V., Ng, C.K., Shao, X., Wang, L.: What causes scatter-free transport of non-relativistic solar electrons? Astrophys. J. 728, 133 (2011). https://doi.org/10.1088/0004637X/728/2/133

Terasawa, T., Oka, M., Nakata, K., Keika, K., Nosé, M., McEntire, R.W., Saito, Y., Mukai, T.: Cosmic-ray-mediated' interplanetary shocks in 1994 and 2003. Adv. Space Res. 37, 1408 (2006). https://doi.org/10.1016/j.asr.2006.03.012

Vestrand, W.T., Forrest, D.J.: Evidence for a spatially extended component of gamma rays from solar flares. Astrophys. J. Lett. 409, L69 (1993). https://doi.org/10.1086/186862

Wiedenbeck, M.E., Cohen, C.M.S., Cummings, A.C., de Nolfo, G.A., Leske, R.A., Mewaldt, R.A., Stone, E.C., von Rosenvinge, T.T.: Persistent energetic ${ }^{3} \mathrm{He}$ in the inner heliosphere. Proc. 30th Int. Cos. Ray Conf. (Mérida). 1, 91 (2008)

Zank, G.P., Rice, W.K.M., Wu, C.C.: Particle acceleration and coronal mass ejection driven shocks: a theoretical model. J. Geophys. Res. 105, 25079 (2000). https://doi.org/10.1029/ 1999JA000455

Zank, G.P., Li, G., Florinski, V., Hu, Q., Lario, D., Smith, C.W.: Particle acceleration at perpendicular shock waves: model and observations. J. Geophys. Res. 111, A6108 (2006). https://doi. org/10.1029/2005JA011524

Zank, G.P., Li, G., Verkhoglyadova, O.: Particle acceleration at interplanetary shocks. Space Sci. Rev. 130, 255 (2007). https://doi.org/10.1007/s11214-007-9214-2

Open Access This chapter is licensed under the terms of the Creative Commons Attribution 4.0 International License (http://creativecommons.org/licenses/by/4.0/), which permits use, sharing, adaptation, distribution and reproduction in any medium or format, as long as you give appropriate credit to the original author(s) and the source, provide a link to the Creative Commons license and indicate if changes were made.

The images or other third party material in this chapter are included in the chapter's Creative Commons license, unless indicated otherwise in a credit line to the material. If material is not included in the chapter's Creative Commons license and your intended use is not permitted by statutory regulation or exceeds the permitted use, you will need to obtain permission directly from the copyright holder. 